\title{
Functional genomics identifies new synergistic therapies for retinoblastoma
}

\author{
Arthur Aubry $^{1,2} \cdot$ Joel D. Pearson ${ }^{1,2,3} \cdot$ Katherine Huang ${ }^{1} \cdot$ Izhar Livne-bar $^{3,4} \cdot$ Mohammad Ahmad $^{1}$. \\ Madhavan Jagadeesan ${ }^{5,9} \cdot$ Vikas Khetan $^{6} \cdot$ Troy Ketela $^{7} \cdot$ Kevin R. Brown $\mathbb{1}^{7} \cdot$ Tao $\mathrm{Yu}^{1} \cdot$ Suying Lu ${ }^{1}$. \\ Jeffrey L. Wrana ${ }^{1,8} \cdot$ Jason Moffat $\mathbb{1}^{7,8} \cdot \operatorname{Rod}$ Bremner $\mathbb{B}^{1,2,3}$
}

Received: 23 March 2020 / Revised: 3 June 2020 / Accepted: 12 June 2020 / Published online: 22 June 2020

(c) The Author(s) 2020. This article is published with open access

\begin{abstract}
Local intravitreal or intra-arterial chemotherapy has improved therapeutic success for the pediatric cancer retinoblastoma (RB), but toxicity remains a major caveat. RB initiates primarily with RBI loss or, rarely, MYCN amplification, but the critical downstream networks are incompletely understood. We set out to uncover perturbed molecular hubs, identify synergistic drug combinations to target these vulnerabilities, and expose and overcome drug resistance. We applied dynamic transcriptomic analysis to identify network hubs perturbed in RB versus normal fetal retina, and performed in vivo RNAi screens in $R B 1^{\text {null }}$ and $R B 1^{w t} ; M Y C N^{a m p}$ orthotopic xenografts to pinpoint essential hubs. We employed in vitro and in vivo studies to validate hits, define mechanism, develop new therapeutic modalities, and understand drug resistance. We identified BRCA1 and RAD51 as essential for RB cell survival. Their oncogenic activity was independent of BRCA1 functions in centrosome, heterochromatin, or ROS regulation, and instead linked to DNA repair. RAD51 depletion or inhibition with the small molecule inhibitor, B02, killed RB cells in a Chk1/Chk2/p53-dependent manner. B02 further synergized with clinically relevant topotecan (TPT) to engage this pathway, activating p53-BAX mediated killing of RB but not human retinal progenitor cells. Paradoxically, a B02/TPT-resistant tumor exhibited more DNA damage than sensitive RB cells. Resistance reflected dominance of the p53-p21 axis, which mediated cell cycle arrest instead of death. Deleting p21 or applying the BCL2/BCL2L1 inhibitor Navitoclax re-engaged the p53-BAX axis, and synergized with B02, TPT or both to override resistance. These data expose new synergistic therapies to trigger p53-induced killing in diverse RB subtypes.
\end{abstract}

\section{Introduction}

Retinoblastoma (RB) is an aggressive cancer of the infant retina initiated by homozygous $R B 1$ tumor suppressor gene inactivation or, rarely, by $M Y C N$ amplification [1-3].

Supplementary information The online version of this article (https:// doi.org/10.1038/s41388-020-1372-7) contains supplementary material, which is available to authorized users.

Rod Bremner

bremner@lunenfeld.ca

1 Lunenfeld Tanenbaum Research Institute, Mount Sinai Hospital, Sinai Health, Toronto, ON, Canada

2 Department of Laboratory Medicine and Pathobiology, University of Toronto, Toronto, ON, Canada

3 Department of Ophthalmology and Vision Science, University of Toronto, Toronto, ON, Canada

4 Krembil Research Institute, University Health Network, Toronto, ON, Canada
Survival, salvaging the eye and preserving vision depend on disease severity at diagnosis and treatment efficacy. Standardized protocols to prevent tumor spread after intravitreal (IVT) injection have been developed, and improved outcomes have led to adoption of this treatment modality in multiple centers $[4,5]$. Intra-arterial chemotherapy has also improved outcome and in advanced cases, alternating this approach with IVT chemotherapy has shown promise without systemic chemotherapy, including for advanced unilateral RB [6, 7]. Notably, combining intra-arterial, IVT

Vision Research Foundation, Sankara Nethralaya, Chennai, India

6 Department of Vitroretina and Ocular Oncology, Sankara Nethralaya, Chennai, India

7 Donnelly Centre, University of Toronto, Toronto, ON, Canada

8 Department of Molecular Genetics, University of Toronto, Toronto, ON, Canada

9 Present address: Dualhelix Genetic Diagnostics, Chennai, India 
and periocular chemotherapy can reduce the time to tumor regression and reduce recurrence in tumors that present with vitreous seeding [8]. Local drug delivery considerably reduces systemic toxicity, however, eye toxicity has been observed with current agents [4, 9]. Thus, innovative therapeutics to improve safety and efficacy are urgently needed. Also, new studies are required to deduce whether $R B 1^{\text {null }}$ and "MYCN ${ }^{a m p}$ " tumors share similar vulnerabilities.

Precision medicine targets activated oncoproteins, but deleted tumor suppressor genes, such as $R B 1$, are not amenable to this approach, and RB tumors exhibit few other mutations [1-3]. It is thus critical to identify key hubs in RB effector networks. MDM2, for example, is expressed in cone precursors, the cell-of-origin of $\mathrm{RB}$, and constrains p53, which is wild type in this cancer [10-12]. Inhibitors targeting MDM2 or its upstream regulators show promise in preclinical studies [13-15]. SKP2, a component of the $\mathrm{SCF}^{\mathrm{SKP} 2}$ ubiquitylating complex, is another promising hub as its loss is synthetic lethal in many $R B 1$ null contexts, including RB [16]. Indeed, blocking activation of the $\mathrm{SCF}^{\mathrm{SKP} 2}$ complex with the neddylation inhibitor MLN4924 (Pevonedistat) shows promise as a new RB therapy [17]. Such studies illustrate the value in dissecting networks that drive RB cell growth and survival to identify novel therapeutic strategies.

The deployment of RNAi and CRISPR/Cas9 libraries has revolutionized the discovery of cancer drivers and drug resistance mechanisms [18-20]. Genome-wide screens are feasible in vitro, but in vivo studies typically require more focused libraries. To identify high value candidates for in vivo screens, we employed Dynamic Network Modularity (DyNeMo). This tool combines transcriptomic and protein network information to define whether the stoichiometry of co-expressed hubs and partners is altered in cancer vs. normal cells. Previously, DyNeMo pinpointed disrupted hubs influencing outcome in breast cancer [21]. Applying this approach to RB transcriptome data, we identify candidates, establish hits through in vivo RNAi screens in $R B 1^{\text {null }}$ and $M Y C N^{\text {amp }}$ tumors, and exploit those insights to develop several drug combinations that synergistically kill RB. Moreover, we identify a resistance mechanism and a strategy to resensitize affected RB cells.

\section{Results}

\section{In vivo screens highlight DNA-repair hubs as drivers in $R B 1^{\text {null }}$ and $R B 1^{\text {wt }} ; M Y C N^{a m p}$ retinoblastoma}

To select candidates for in vivo shRNA screens we applied DyNeMo [21]. It correlates transcriptional co-expression of hubs (proteins with $>4$ known partners) and their partners in two conditions (e.g., normal vs. cancer), exposing hubs where these correlations differ. Thus, absolute expression is not relevant but rather the level of network components relative to one another. Using transcriptome data from 21 human $R B 1^{\text {null }}$ tumors, and 12 human fetal retinal samples, we identified 27 disrupted hubs (Fig. 1a, b, Fig. S1A, B, Table S1 "DyNeMo result"). Hits were enriched in DNArepair factors, including BRCA1, RAD51, and XRCC6 (Gene Ontology analysis, $p=0.031$ ), and the BRCA1 partner PABPC1. We also assessed orthologous mouse genes from 6 mouse RB models (see "Materials and methods"), which confirmed 11 hubs (Fig. 1a, b, Fig. S1A, B, Table S1 "DyNeMo result").

We performed in vivo functional shRNA screens with the 27 disrupted hubs and 34 selected tumor suppressors and oncogenes including positive controls known to drive RB (Fig. 1a, b, Table S1 "gene list 1st screen"). We also selected undisrupted hubs and genes with equal expression in tumor vs. human fetal retina (Table S1 "gene list 1st screen"). In total, 647 shRNAs targeting 147 genes were tested. Y79 and WERI-RB1 RB cells were transduced with bar-coded lentiviral shRNAs, drug selected, and genomic (g) DNA collected immediately (time zero (T0)) or from tumors grown from orthotopically transplanted cells (Fig. 1c, and "Materials and methods"). Six tumors per cell line were deep sequenced to identify dropouts (see "Materials and methods", Fig. S2, Table S1 "1st screen normalized reads"). Of 647 shRNAs, 72 and 100 were significantly depleted in WERI-RB1 and Y79 tumors, respectively (Fig. 1d, $n=6, Z$-score $<-1.96, p<0.05$ ). In total, 18 genes had $\geq 2$ significantly depleted shRNAs, of which 9 scored in both lines, while 6 and 3 scored only in Y79 or WERI-RB1, respectively (Fig. 1d, Table S1 "in vivo Zscore 1st screen").

To identify robust and broadly relevant hits, a second iterative in vivo screen was performed with WERI-RB1 and Y79, but also RB3535S, a low passage $R B 1^{\text {null }} \mathrm{RB}$ cell line, and RB3823, derived from rare $R B 1^{w t} ; M Y C N^{a m p} \mathrm{RB}$ [3]. In total, 138 shRNAs/53 genes were processed including: 55 high scoring shRNAs/18 genes from the primary screen; 9 shRNAs/3 borderline genes (defined in Table S1 "2nd screen normalized reads" and "summary table 1st 2nd screen"); negative controls, including 55 shRNA/18 gene non-hits from the first screen, and 4 irrelevant shRNA targets (GFP, RFP, LacZ, luciferase), and; positive controls targeting 12 broadly essential genes [18] (Fig. 1e, Table S1 "2nd screen normalized reads" and "summary table 1st 2nd screen"). The shRNAs were well-represented at T0 as $\geq 98 \%$ yielded $\geq 10$ normalized reads in all 4 lines (Fig. S3A). T0 samples were highly correlated, 5/6 WERI-RB1 and 6/6 Y79 tumors formed one cluster, and 6/6 RB3823 and 5/6 RB3535S tumors formed a second cluster (Fig. S3B). 
A

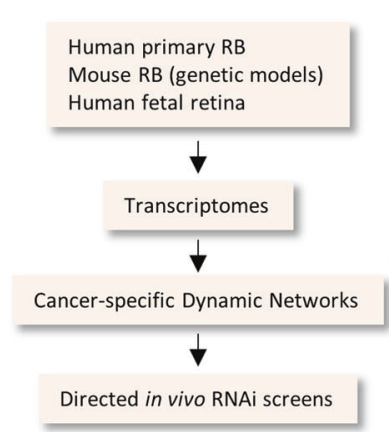

B

\begin{tabular}{lccc}
\multicolumn{1}{c}{ Category } & \# $\begin{array}{c}\text { \# of } \\
\text { genes }\end{array}$ & $\begin{array}{c}\text { \# of } \\
\text { shRNAs }\end{array}$ \\
\hline Hum+Mouse Disrupted Hubs & 1 & 11 & 47 \\
Human Disrupted Hubs & 2 & 16 & 73 \\
Selected Tum Supp-Onc & 3 & 34 & 175 \\
Random Control Hubs & 4 & 28 & 115 \\
Unchanged Controls & 5 & 58 & 237 \\
\hline \multicolumn{1}{c}{ Total } & & 147 & 647
\end{tabular}

D

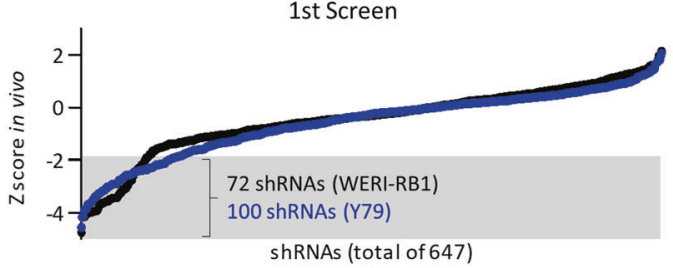

E

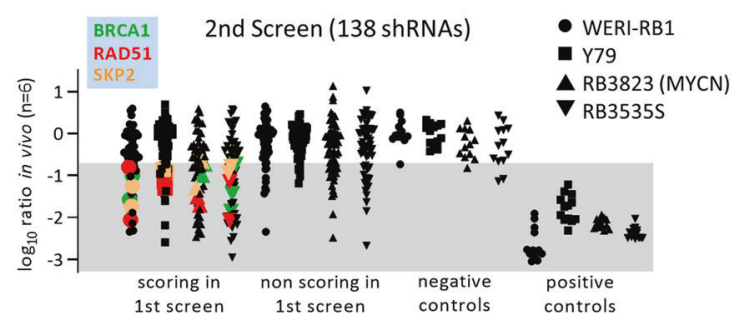

Human fetal retina
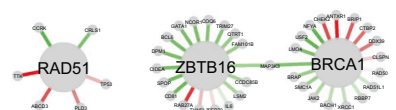

SRF

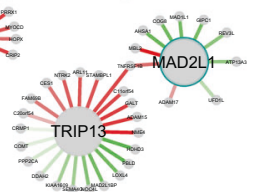

PABPC1

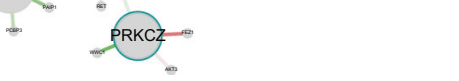

coexpression disrupted hub in Human RB

disrupted hub in Human+Mouse RB

- hub partner
Retinoblastoma
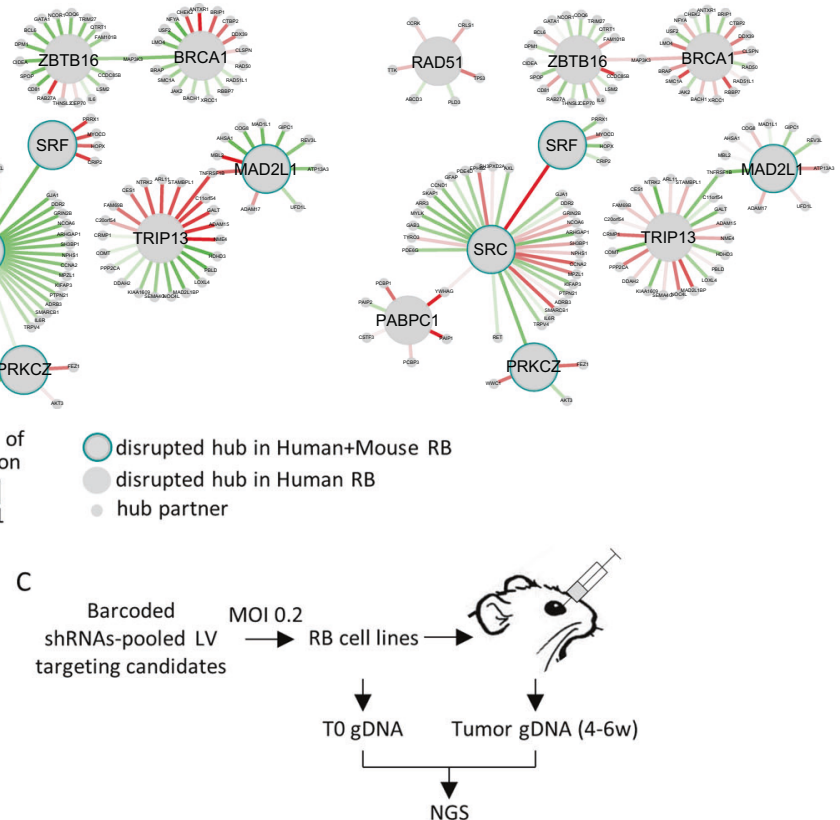

shRNA depletion (Tumor/TO)

$\mathrm{F}$

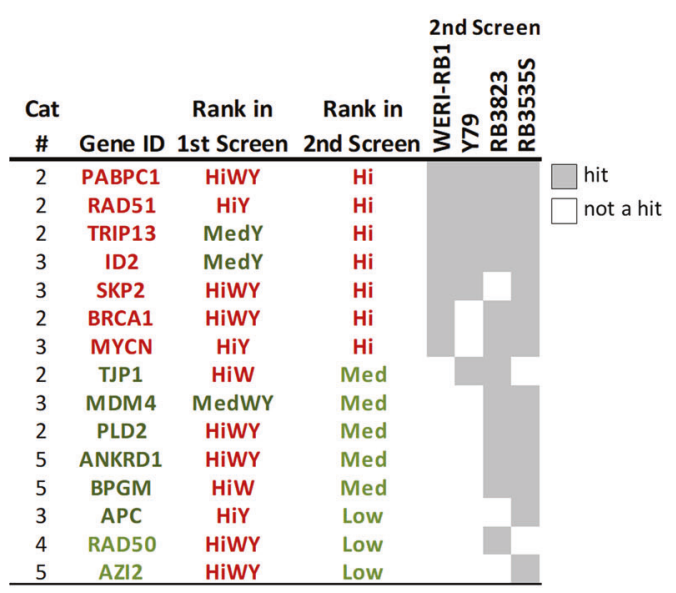

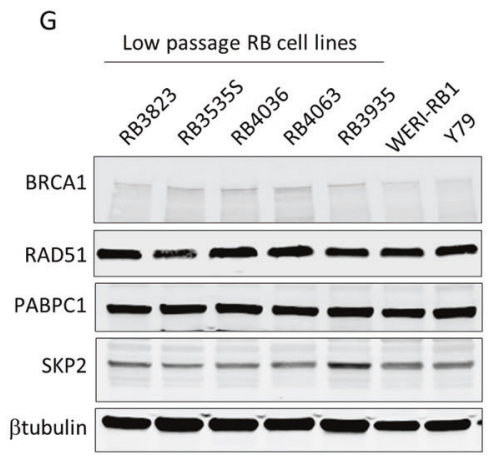

$\mathrm{H}$
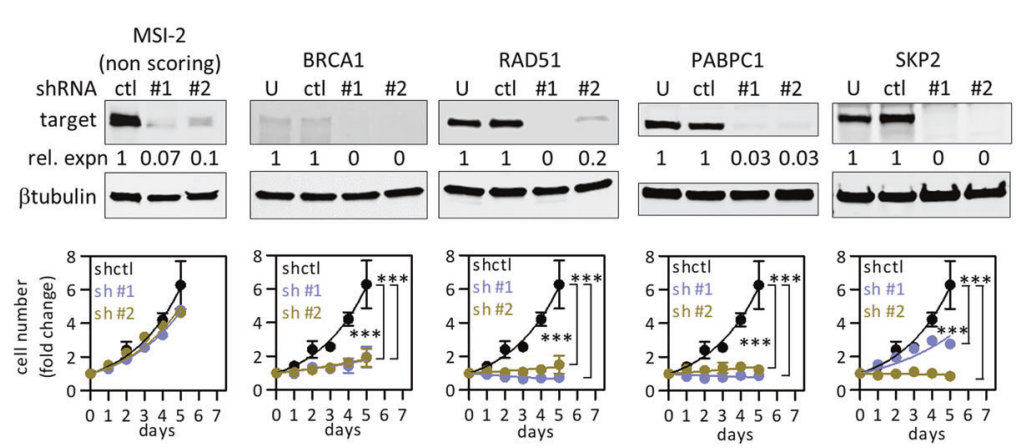

SKP2

Tumor growth was inhibited by $13 / 13$ positive (essential gene) but $0 / 4$ negative control shRNAs, validating the approach (Fig. 1e). 15/18 (83\%) hits from the first screen

were validated in the second screen, while only $3 / 18$ non-hit controls from the first screen scored in the second ( $p=$ 0.0002 , Fisher's exact test) (Fig. 1e, Table S1 "ratio tumor 
Fig. 1 In vivo RNAi screens expose retinoblastoma vulnerabilities. a Strategy to identify novel therapeutic targets in RB. Examples of disrupted hub/partners identified by DyNeMo are shown. b Sources of the 647 shRNAs targeting 147 genes for the primary in vivo dropout screen. $\mathbf{c}$ Design of the in vivo shRNA screens. d Primary screen data from orthotopic RB xenografts. shRNA enrichment/depletion was determined from $Z$-scores (full dataset in Table S1). Shaded area represents significant dropouts $(Z<-1.96, p<0.05$ two tails). e Secondary screen data. In total, 138 shRNAs selected from the primary screen were tested in the indicated four orthotopic RB xenografts. The averaged $\log$ ratio tumor/T0 reads $(n=6)$ was plotted. Shaded area represents dropouts (full dataset in Table S1). The recurrent hits BRCA1, RAD51, and SKP2 are highlighted. f Summary of hits from the primary and secondary in vivo dropout screens. Hi: high rank; Med: medium rank; W: WERI-RB1; Y: Y79; red: high rank; dark green: medium rank; light green: low rank. $\mathrm{g}$ Westerns showing expression of the main hits BRCA1, RAD51, PABPC1, SKP2 in multiple RB lines. h Screen validation. Y79 cells were transduced with individual shRNAs of the indicated hits or a nonscoring control, knockdown efficiency assessed by western blot, and the effect on growth measured over 5 days by CellTiter-Glo reagent. Data were normalized to $\mathrm{d} 0$ and plotted $(n=3$, mean $\pm \mathrm{SD}$, *** $p<0.001$ twoway ANOVA, Sidak's multiple comparisons test).

vs. T0 2nd screen" and "summary table 1st 2 nd screen"). Hits were classified as high, medium, or low quality if they scored in $\geq 3,2$, or 1 of the 4 cell lines, respectively (Fig. 1f, Table S1 "summary table 1st 2nd screen"). Seven genes were hits in 3/4 or 4/4 lines, indicating good concordance and shared networks across $R B^{\text {null }}$ and $M Y C N^{a m p}$ cell lines. These included SKP2 or MYCN (Fig. 1f), confirming prior data $[10,16]$, and ID2, which drives oncogenesis in other $R B 1^{\text {null }}$ and $R B 1^{w t} / M Y C N^{a m p}$ contexts, including neuroblastoma [22-24]. Paradoxically, ID2 is a tumor suppressor in SV40 large T-driven murine RB [25], so this model does not mimic human RB in this regard. Several highly ranked hits were linked to DNA repair, including RAD51 and BRCA1, which cooperate to mediate homologous recombination (HR) [26], PABPC1, which binds BRCA1 [27], and TRIP13, which promotes DNA repair [28, 29]. All four were hits in both DyNeMo and functional screens, and TRIP13 binds the NHEJ protein XRCC6 (KU80), which DyNeMo flagged as a disrupted hub (Fig. 1a, Fig. S1A, B). We confirmed expression of BRCA1, RAD51, and PABPC1 in multiple low passage RB cell lines, and depletion in Y79 cells, like that of SKP2 but not a negative control locus MSI2, severely inhibited growth in vitro (Fig. 1g, h). Doxycyclin-inducible re-expression of RAD51 further validated the on-target effect of siRAD51 (Fig. S4). Thus, DyNeMo identified relevant targets, exposing overlapping oncogenic networks in $R B I^{\text {null }}$ and $R B I^{w t} / M Y C N^{a m p}$ $\mathrm{RB}$, and essential roles for DNA-repair proteins in both tumor subtypes. The retinoblastoma protein $(\mathrm{pRB})$ can promote HR and NHEJ [30-32], RB1 loss is linked to increased DNA damage [33-36], and the combination of HR gene and $R B 1$ mutations is beneficial in ovarian cancer
[37], but whether directly targeting these repair processes could enhance RB treatment is unclear.

\section{Sensitivity to BRCA1 independent of non-DNA- repair functions}

The pro-tumorigenic role of BRCA1 "tumor suppressor" in $\mathrm{RB}$ is intriguing. In addition to DNA repair (see below), there have been claims that it regulates centrosome duplication, heterochromatin integrity and redox [38-40]. In RB cells, BRCA1 foci did not co-localize with pericentrinpositive centrosomes; subcellular fractionation detected BRCA1 in the soluble nuclear compartment but not the cytoplasm where centrosomes are located, and BRCA1 depletion did not alter centrosome number (Fig. S5A-C). BRCA1 loss also did not perturb HP1-positive heterochromatic foci (Fig. S6A, B). Depleting BRCA1 or RAD51 did induce ROS in Y79 cells (Fig. S7A-E). Across four cancer cell lines, increased ROS due to RAD51 depletion correlated with reduced cell numbers, although that was not paralleled in BRCA1-depleted cells (Fig. S7F-H). However, in Y79 or A549 cells, where ROS induction was the greatest, the scavenger trolox reduced ROS, but did not ameliorate G2/M arrest or apoptosis (Fig. S7I-M). Thus, in RB cells BRCA1 does not affect centrosome duplication or heterochromatin stability, and while both BRCA1 and RAD51 suppress ROS, G2/M arrest and cell death induced by their inactivation are ROS independent. Thus, we focussed on DNA damage.

Consistent with induction of DNA breaks in $R B 1^{\text {null }}$ contexts [33-36], $\sim 60 \%$ of untreated $\mathrm{Y} 79$ cells had $\gamma \mathrm{H} 2 \mathrm{AX}$ foci, predominantly in $\mathrm{EdU}^{+} \mathrm{S}$-phase cells (Fig. S8A, B), where BRCA1, RAD51, and $\gamma \mathrm{H} 2 \mathrm{AX}$ foci were also detected (Fig. S8A, B). BRCA1 and RAD51 siRNA hindered Y79 cell growth, which correlated with G2/M arrest and apoptosis, evident from subG1 cells, DNA fragmentation, and PARP cleavage (Fig. 2a-d, Fig. S9A, F, G). Depleting RAD51 had a similar effect in another cell line, RB1021 (Fig. 2a-d, Fig. S9B). Micronuclei suggested mitotic stress following DNA damage (Fig. S9D, E). Depleting BRCA1 or RAD51 induced $\gamma \mathrm{H} 2 \mathrm{AX}$, p53 expression, DNA damage-related p53 activation (Ser15 phosphorylation), and the p53-target gene p21 (Fig. 2d). The degree of apoptosis and growth inhibition was slightly less following BRCA1 depletion (Fig. 2a-d, Fig. S9A, F, $\mathrm{G})$, and indeed BRCA1 loss did not completely eliminate RAD51 recruitment to $\gamma \mathrm{H} 2 \mathrm{AX}$ foci (Fig. 2e). The latter might reflect remnant BRCA1 and/or proteins that promote HR in BRCA1-deficient cells [29]. We focussed subsequent assays on RAD51. Immunostaining confirmed $\gamma \mathrm{H} 2 \mathrm{AX}$ induction following RAD51 knockdown, an increased number of foci/cell in both S- and non-S-phase cells, and enhanced foci size in the latter (Fig. 2f, h). Thus, persistent 
A

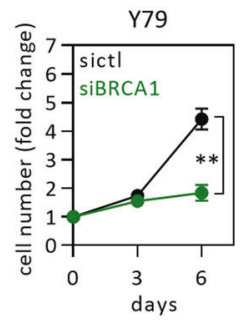

B

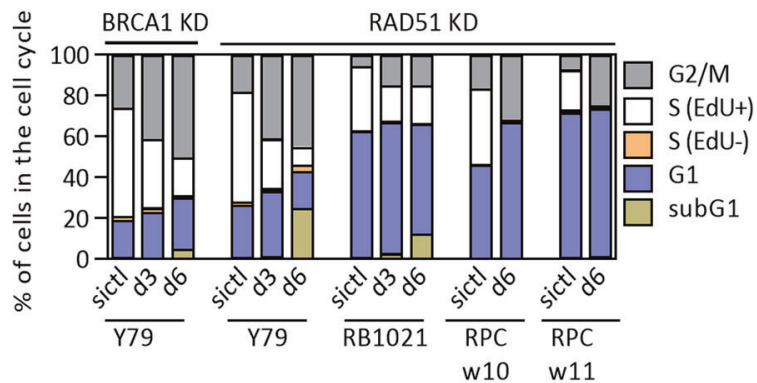

D
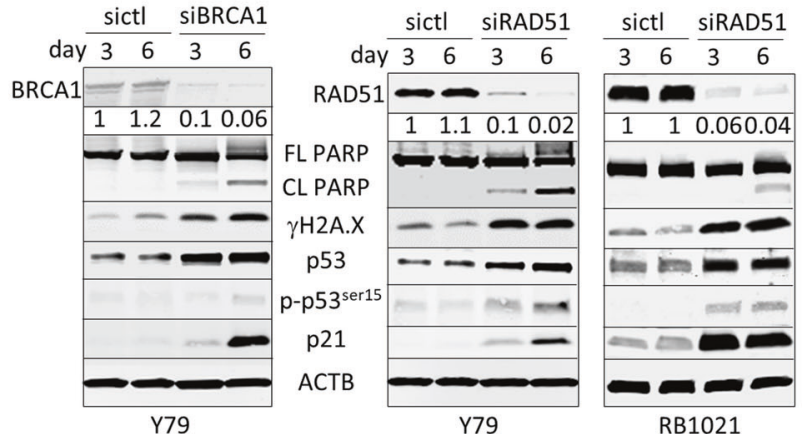

RB1021

G

F

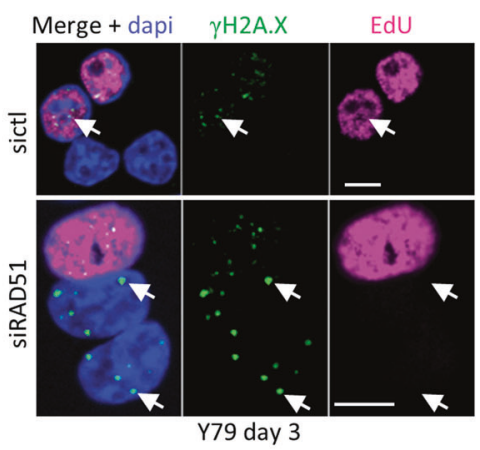

I

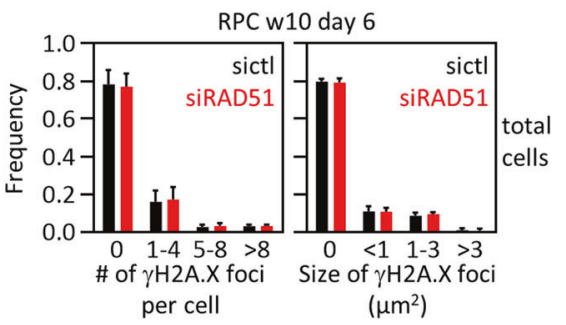

total
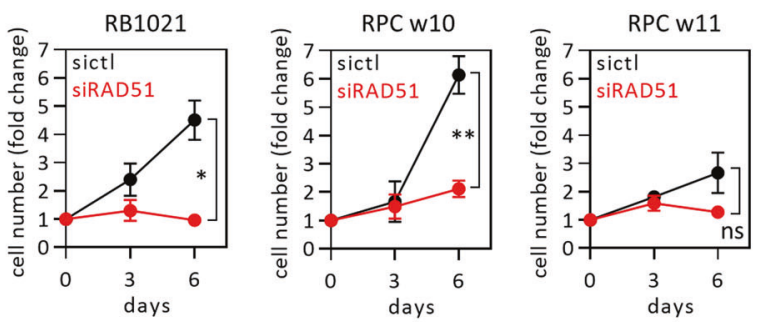

C
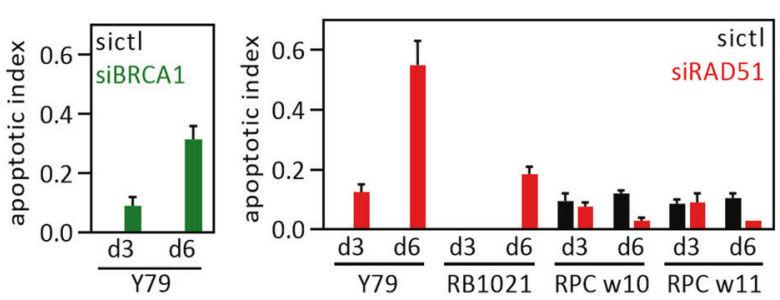

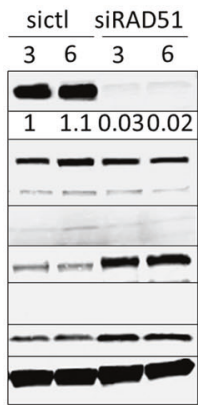

RPC w10

RPC $w 10$ day 6

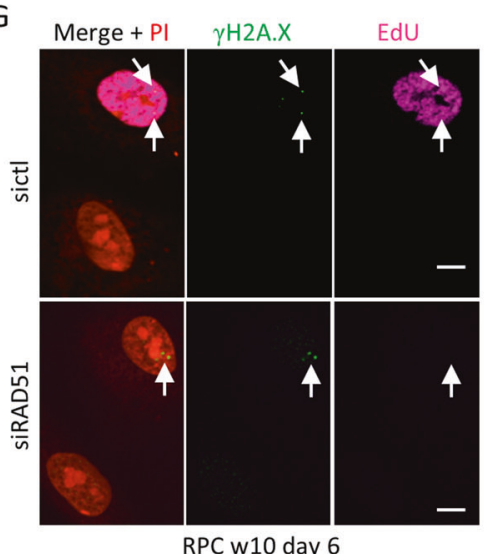

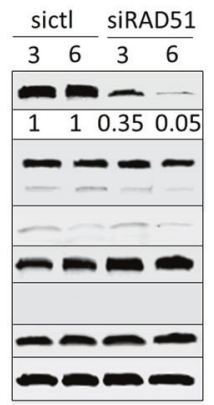

E $\rightleftharpoons$ no RAD51 foci

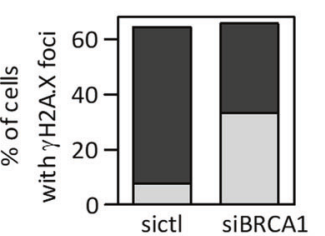

RPC w11

$\mathrm{H}$

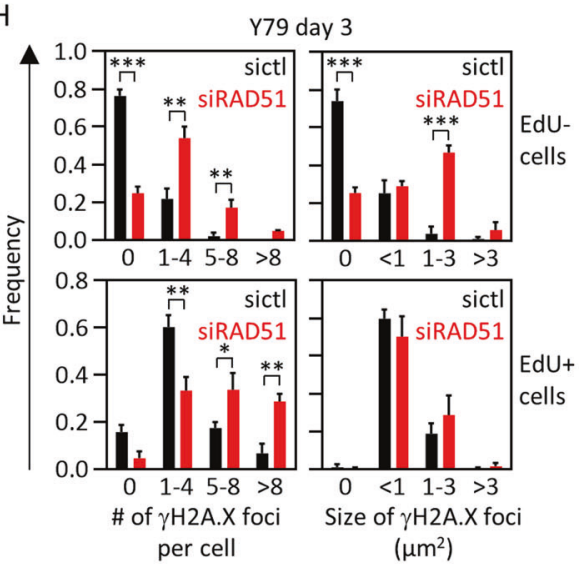


Fig. 2 RAD51 loss kills retinoblastoma but not human fetal retinal cells. a-d The indicated RB tumor cells or RPC were treated with the indicated siRNAs for 6 days, and growth (a), cell cycle phase (b), apoptosis (c) and protein levels (d) determined. Representative flow cytometry plots used for (b) are shown in Fig. S9A-C. Graph in (c) is quantification of PARP cleavage in (d) $(n=2$, mean \pm range). e Quantification of nuclear RAD51 and $\gamma \mathrm{H} 2 \mathrm{~A}$.X foci in Y79 cells treated with siCtl or siBRCA1, detected by immunostaining at day 6 and analyzed by confocal microscopy. f-i Y79 cells (f) or RPC (g) were treated with siCtl or siRAD51, labeled with EdU (magenta) and $\gamma \mathrm{H} 2 \mathrm{~A} . \mathrm{X}$ (green) at the indicated timepoints, and confocal images obtained. Arrows indicate $\gamma \mathrm{H} 2 \mathrm{~A} . \mathrm{X}$ foci. The number and size of $\gamma \mathrm{H} 2 \mathrm{~A} . \mathrm{X}$ foci were quantified in $\mathrm{EdU}^{+}$(S-phase) $\mathrm{EdU}^{-}$(non-S-phase) Y79 cells (h) or all RPC (i). In all cases $n=3$ (unless specified otherwise). Data in (a), (h), (i) indicate mean \pm SD. In (a), $* p<0.05$, $* * p<0.01, \quad * * * p<0.001$, ns nonsignificant, two-way ANOVA, Sidak's multiple comparisons test. In (h) and (i), ${ }^{*} p<0.05, * * p<0.01$, $* * * p<0.001$ Student $t$ test. Scale bars are $10 \mu \mathrm{m}$.

DNA damage correlates with the phenotypic effects of BRCA1/RAD51 depletion in RB cells.

\section{RAD51 depletion kills RB tumor but not human retinal progenitor cells}

Sensitivity to RAD51 depletion might be a general feature of dividing fetal retinal cells rather than a specific property of RB. Human RB arises from ectopically dividing cone precursors but normal cones do not divide, so the closest possible normal cell comparison would be retinal progenitor cells (RPC), from which cones are derived. Thus, we cultured RPC from week 10 (w10) and w11 fetuses. RPC-w 10 grew similarly to RB cells while RPC-w 11 grew more slowly (Fig. 2a). We confirmed that the cells were cycling with Ki67 and cyclin D1, and validated retinal neurogenic progenitor identity with multiple markers (Chx10, Sox2, Neurod1, Pax6); the cells also expressed glutamine synthetase, which is a late progenitor/Muller glia marker, but lacked Gfap ruling out contaminating astrocytes derived from nonretinal cells and implying some differentiation in culture (Fig. S10A-D). Progenitor and Müller glia transcriptomes are 50\% identical [41], thus either provides a reasonable baseline for RAD51 dependence in dividing retinal cells. Assessment of $\gamma \mathrm{H} 2 \mathrm{AX}$ and RAD51 foci revealed basal levels of damage and HR activation in RPC, which was, as expected, mainly in Sphase, and the proportion of affected cells was somewhat lower in RPC vs. RB cells (Fig. S8A, C). RAD51 levels were similarly depleted by siRNA in RPC and RB cells (Fig. 2d), but despite G2/M arrest in both scenarios, p53 activation and persistence of $\gamma \mathrm{H} 2 \mathrm{AX}$ foci, and increases in micronuclei, fragmented DNA, subG1 cells, and PARP cleavage were all unique to RB cells (Figs. $2 b-d, 2 F-I$, Fig. S9A-G). Thus, RAD51 depletion selectively kills RB cells.

\section{CHK1/2 drive cell cycle arrest and p53-mediated apoptosis in HR-depleted RB cells}

Next, we investigated the molecular basis of HR sensitivity. Above, we showed that RAD51 depletion activated p53, and indeed RB cells survived when both RAD51 and p53 were depleted (Fig. 3a-c). Following DNA damage, ATM/ ATR phosphorylate CHK1/2 kinases, which promote degradation of CDC25 phosphatase, causing G2/M arrest [42-44]. Indeed, depleting RAD51 drove phosphorylation of ATM/ATR sites on CHK1 and CHK2 at Ser317 and Thr68, respectively, accompanied by CDC25A downregulation (Fig. 3b). ATM or CHK2 inhibitors blocked p53 and H2AX phosphorylation, preventing PARP cleavage, and while CHK2 inhibition modestly rescued CDCD25A levels, CHK1 inhibitor PF-477736 was more potent (Fig. S11A, B). These data reveal functional relevance for all three kinases in RAD51-depleted RB cells.

Depleting p53 in siRAD51-treated cells did not fully rescue cell number (Fig. 3a). Cell cycle analysis showed that while depleting p53 reduced subG1 cells (confirming PARP analysis), it did not affect G2 arrest (Fig. S11C). CHK1/2 phosphorylate CDC25 proteins after DNA damage [44, 45], and as noted above, CHK inhibitors rescued CDC25A levels, suggesting a possible mechanism for G2 arrest (Fig. S11B). Indeed, treating RAD51-depleted cells with either of two CHK1 inhibitors (PF-477736, MK-8776) or CHK2 inhibitor II reduced G2 cells, and combining MK-8776 with CHK2 inhibitor II potentiated the effect (Fig. S11D). Alone, CHK inhibitors also partially restored the proportion of replicating $\left(\mathrm{EdU}^{+}\right)$cells and total cell number, and rescue was nearly complete when CHK1/2 inhibitors were combined (Fig. S11E, F). Thus, CHK1/2 cooperatively promote $\mathrm{G} 2$ arrest in HR-deficient RB cells, likely through $\mathrm{CDC} 25$, and cell death through p53.

\section{HR-defective RB cells are hypersensitive to topoisomerase inhibitors in a p53-dependent manner}

Combining DNA-damaging agents with DNA-repair inhibitors can improve chemotherapeutic efficacy [46, 47]. This approach has yet to be explored for RB management, even though DNA-damaging agents such as topotecan (TPT), etoposide (ETO), and melphalan are used routinely to reduce tumor load [48]. We asked whether inhibiting HR potentiates TPT and/or ETO.

TPT or ETO induced RAD51 foci in 95 or $60 \%$ of cells, respectively, and RAD51-depleted cells lacked foci, confirming antibody specificity (Fig. S12A, B). Fifty percent of vehicle-treated cells were RAD51 ${ }^{+}$but with $1-3$ foci, whereas $76 \%$ of RAD51 ${ }^{+}$TPT-treated cells had $>12$ foci/ cell, and $63 \%$ of RAD51 ${ }^{+}$ETO-treated cells had 4-9 foci/cell 
A

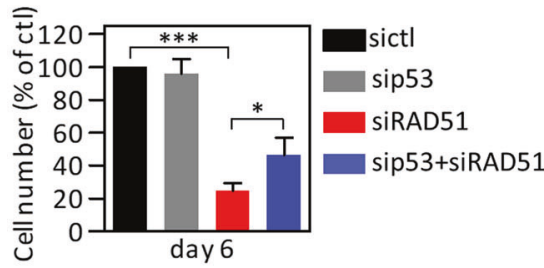

C

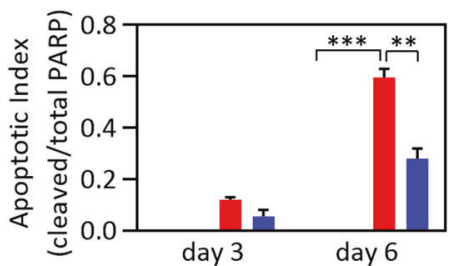

D
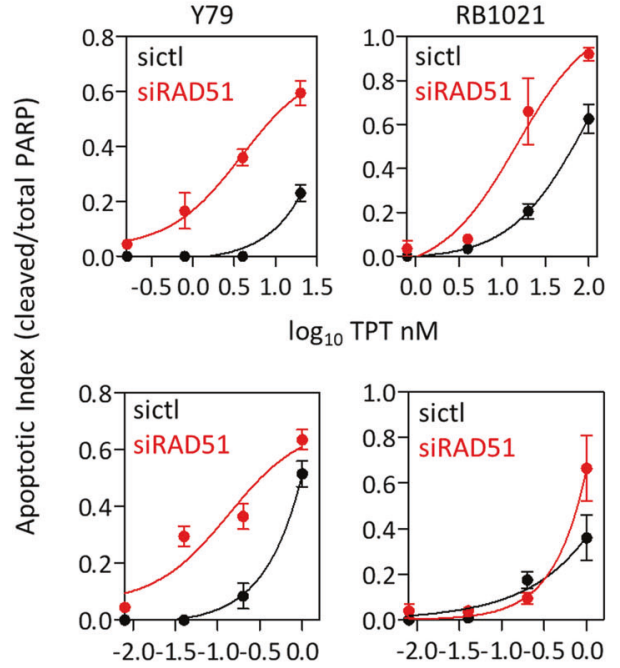

$\log _{10} \mathrm{ETO} \mu \mathrm{M}$

E
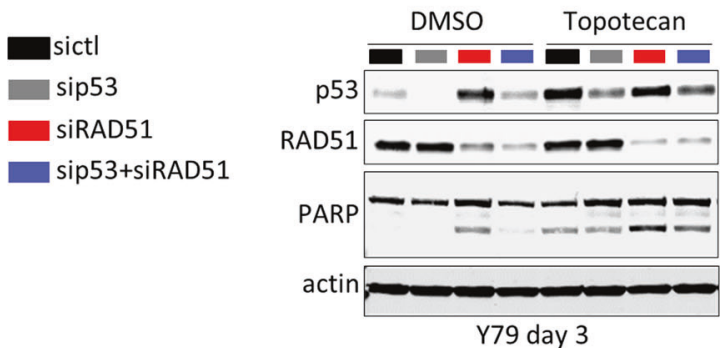

Fig. 3 Depleting RAD51 sensitizes RB cells to topoisomerase inhibitors by promoting p53-mediated death. a-c Y79 cells were treated with the indicated siRNAs for 3 or 6 days (nM indicated in (b)) and growth (trypan blue counts, (a)), protein levels (westerns, (b)), and apoptosis (PARP cleavage, $(\mathbf{c}))$ assessed. $(n=3$, mean $\pm \mathrm{SD}, * p<$ $0.05, * * p<0.01, * * * p<0.001$ ordinary one-way ANOVA, Tukey's multiple comparisons test). d Y79 or RB1021 cells were treated with

(Fig. S12A-C). RAD51 foci overlapped $\gamma \mathrm{H} 2 \mathrm{~A} . \mathrm{X}$ in all drug treatments, demonstrating coincident DNA damage and HR (Fig. S12A). Consistent with previous work, TPT and ETO

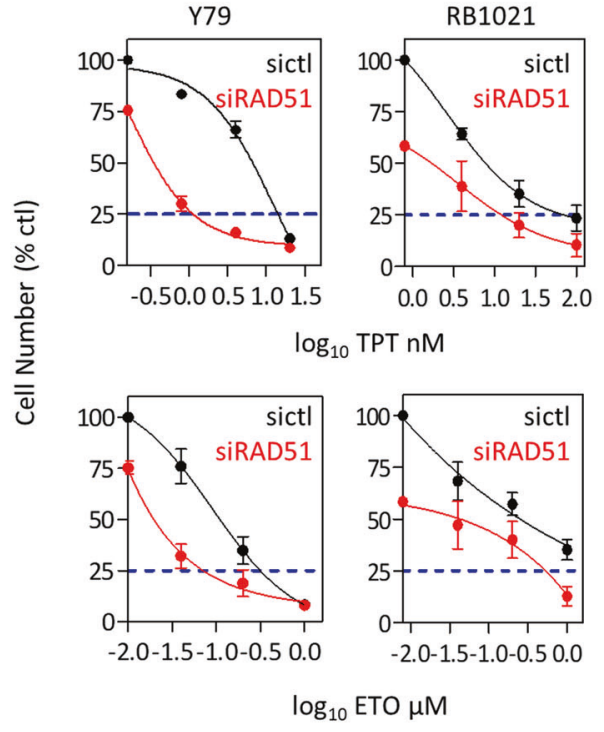

$\mathrm{F}$

Cell Number $(\% \mathrm{ctl})$

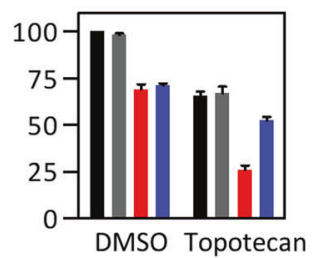

G

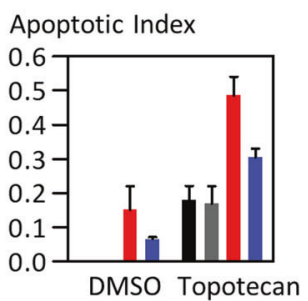

siCtl or siRAD51 plus either TPT or ETO, and dose-response curves obtained for cell number (trypan blue) or apoptosis (PARP cleavage, representative blot in Fig. S13A). ( $n=3$, mean \pm SD). e-g Y79 cells were treated with siRNAs and drugs as indicated. At day 3 , cells were harvested for westerns (e), cell counts (f), or apoptosis (PARP cleavage, $(\mathbf{g}))(n=2$, mean \pm range $)$.

induced potent G2 arrest (Fig. S12D) [49, 50]. High throughput analysis of $\gamma \mathrm{H} 2 \mathrm{~A} . \mathrm{X}$ foci in the cell cycle with imaging flow cytometry (ImageStream ${ }^{\circledR} \mathrm{X}$ ) confirmed that 
TPT blocked cells in $\mathrm{G} 2$ due to extensive DNA damage in $\mathrm{S}$ + G2 (Fig. S12E-I). Thus TPT and ETO induce DNA damage and activate RAD51 in RB cells.

Next, Y79 and RB1021 cells were treated for $72 \mathrm{~h}$ with control or RAD51 siRNA (efficiency $>90 \%$, Fig. S13A) plus either DMSO or increasing doses of TPT or ETO. Depleting RAD51 enhanced apoptosis in response to multiple concentrations of either drug in Y79 cells and similarly for TPT in RB1021 cells, although for unclear reasons sensitivity was elevated only at the highest dose of ETO in RB1021 cells (Fig. 3d, Supplementary Fig. S13). In Y79 cells, siRAD51 reduced TPT EC75 16-fold (Control $13.5 \mathrm{nM}$ vs. siRAD51 $0.84 \mathrm{nM}$ ), or 11 -fold for ETO (330 nM vs. $32 \mathrm{nM}$ ), and 13-fold or 4-fold in RB1021, respectively (Fig. 3d). PARP cleavage and annexin $\mathrm{V}$ staining confirmed increased apoptosis (Fig. 3d, Fig. S13A, B). For example, the apoptotic index with $20 \mathrm{nM}$ TPT in Y79 cells was reached with only $0.8 \mathrm{nM}$ following RAD51 depletion, a 25-fold reduction. Enhanced cell death was p53 dependent (Fig. 3e-g). Thus, disrupting HR sensitizes RB cells to clinically relevant topoisomerase inhibitors.

\section{A small molecule RAD51 inhibitor synergizes with topo inhibitors to promote apoptosis in RB cells}

Thus far, our network analysis and RNAi screens link HR to RB cell survival. Depleting RAD51 selectively kills RB compared to fetal retinal cells, through activation of a CHK1/2-p53 DNA-damage response pathway. Moreover, RAD51 knockdown sensitizes RB cells to clinically relevant chemotherapeutic drugs. Next, therefore, we tested whether a small molecule inhibitor of RAD51 polymerization, B02 [51], could also synergistically kill RB cells. This agent enhances cisplatin, but only modestly enhances ETO and TPT efficacy in breast cancer MDA-MB-231 cells [52]. B02 dose-response curves on three RB lines revealed EC50s of 15-20 $\mu \mathrm{M}$ (Fig. S14A), comparable to other cell lines [52]. In soft agar, B02 impaired Y79 colony formation with an EC50 of $7 \mu \mathrm{M}$ (Fig. S14B). Cells treated with $25 \mu \mathrm{M}$ for $48 \mathrm{~h}$ lacked RAD51 foci (Fig. S14C). Like RAD51 depletion, B02 caused G2/M arrest, elevated DNA damage, and at higher concentrations $(25 \mu \mathrm{M})$ induced p53 activation and apoptosis in Y79 cells (Fig. S14D-F). RB cells were twice as sensitive as RPC (EC50 15 vs. $30 \mu \mathrm{M}$, Fig. S14A). Cancer selectivity was retained even after longterm exposure (9 days) (Fig. S14G-I). Thus, RAD51 depletion or inhibition is selectively lethal.

B02, like siRAD51, also enhanced TPT and ETO toxicity (Fig. S15A, B). To assess synergy, we determined the Combination index $(\mathrm{CI})$ for growth inhibition or apoptosis (fractions affected $(\mathrm{Fa})$ ), then plotted Fa vs. CI for each combo concentration [53]. Several combos were synergistic $(\mathrm{CI}<1)$ in both $\mathrm{Y} 79$ and RB1021, for both drug combinations, and some were hypersynergistic $(\mathrm{CI}<0.7$, $\mathrm{Fa}>0.7$ ) (Fig. 4a). Multiple B02 + TPT combos also synergistically impaired 3D colony growth of Y79 cells (Fig. S15C, D). Like siRAD51+TPT (Fig. 3e-g), killing by $\mathrm{B} 02$ + TPT was p53-dependent, as CRISPR-mediated p53deletion rendered $\mathrm{RB} 1021$ resistant to $\mathrm{B} 02$ alone or $\mathrm{B} 02 /$ TPT (Fig. 4b, c, Fig. S16A). BAX is a well-known proapoptotic p53 target [54], constrained by antiapoptotic proteins such as BCL2L1 (BCL-XL) [55], and the BAX/ BCL2L1 ratio was increased by TPT, and more-so the combo, all of which was p53-dependent (Fig. S16A). Moreover, with the same conditions and cutoffs there was no B02 + TPT synergy in p53-mutant MDA-MB-231 cells (Fig. S16B, C). Synergistic death in RB cells was confirmed by increased apoptotic morphology and Annexin V/Fxcycle Violet staining (Fig. S16D, E). In contrast, drug combos did not affect RPC (Fig. 4d, Fig. S16F, G). Thus, B02 recapitulates RAD51 depletion, including cancer-specific p53dependent synergy with TPT and ETO.

\section{B02 and TPT synergize in vivo}

Next, we assessed in vivo efficacy. It is important to define suboptimal drug doses for synergy studies. A single IVT injection of $\mathrm{B} 02$ revealed dose and time dependent responses in three orthotopic $\mathrm{RB}$ xenograft models (Fig. S17A-C). By day 7, $30 \mu \mathrm{g}$ B02 inhibited Y79, RB1021, and WERI-RB1 tumors 72\%, 85\%, and 50\%, respectively, reduced division (EdU, Ki67) and increased apoptosis (Active Caspase 3 (AC3)) without affecting retinal morphology (Fig. S17D, E). Thus, B02 is a potentially novel RB therapeutic. IVT TPT is effective in human vitreal disease [48], but in preclinical models IVT delivery has only been tested on retinal disease [56]. In vivo analysis showed dose-responsiveness in all three RB models without toxicity up to $1 \mu \mathrm{g}$ (Fig. S17B, C, E). After defining subEC50 doses, we next tested synergy. IVT B02 + TPT inhibited Y79 and RB1021 tumors better than either drug alone (Fig. 4e-g), matching in vitro data. At endpoint (day 7 ), the combo inhibited Y79 or RB1021 tumor growth $70 \%$ or $85 \%$ vs. $35 \%$ or $50 \%$ with single drug, respectively (Fig. 4f, g). Dose reduction index (DRI) compares the amount of single drug required to achieve the effect with the amount used in the combo. The in vivo calculated DRIs for Y79 were $3.4(10 \mu \mathrm{g}$ vs. $34 \mu \mathrm{g})$ for B02, and $13(0.001 \mu \mathrm{g}$ vs. $0.013 \mu \mathrm{g}$ ) for TPT, while for RB1021 they were 5.4 (3 $\mu \mathrm{g}$ vs. $16 \mu \mathrm{g})$ for B02 and $244(0.001 \mu \mathrm{g}$ vs. $0.24 \mu \mathrm{g})$ for TPT (Fig. 4f). CIs were 0.39 in Y79 and 0.19 in RB1021, indicating potent synergy in both RB tumors (Fig. 4f). Unexpectedly, a similar trial with WERI-RB1 revealed a CI $>1$, indicating antagonism (Fig. 4f). Thus, HR inhibition potently synergizes with TPT in a subset of RB tumors, offering a strategy to reduce toxicity with IVT delivery [57]. 
A
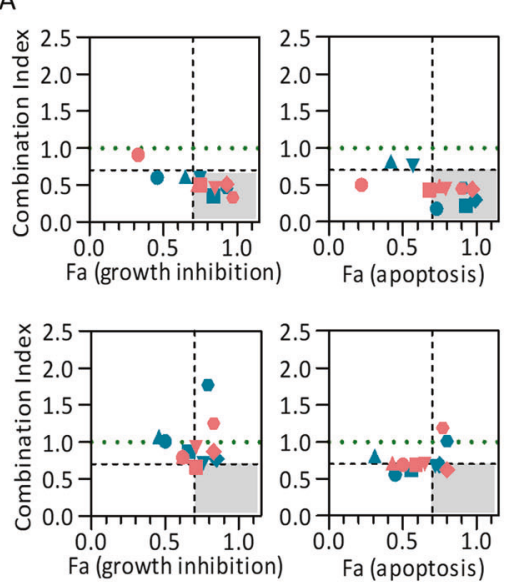

RB1021

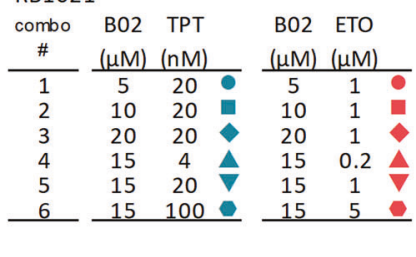

$E$

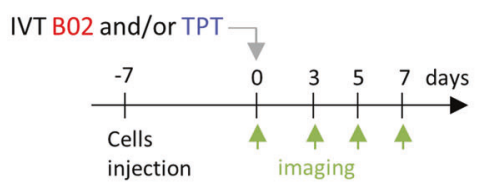

G

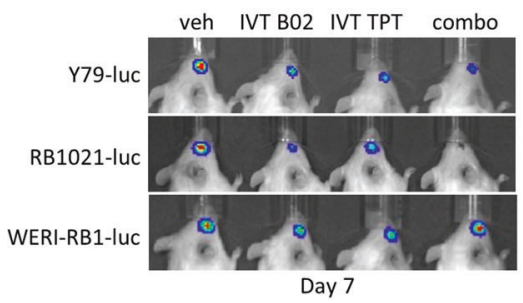

Fig. 4 p53-dependent and tumor-selective synergy of $\mathrm{BO2}$ with standard RB chemotherapy. a Combination Index (CI) vs. effect (Fa) plots for two RB cell lines treated with the indicated two-drug combos of B02, topotecan (TPT), and etoposide (ETO). Turquoise and pink data points show $\mathrm{B} 02+$ TPT and $\mathrm{B} 02+$ ETO combos, respectively. The gray area delineates potent synergistic combos $(\mathrm{CI}<0.7$ and $\mathrm{Fa}>0.7$ ), and the green dotted line $\mathrm{CI}=1$; detailed growth inhibition curves, PARP westerns, and quantification curves are shown in Fig. S15A, B. b, c RB1021 cells were transduced with control or p53 sgRNA lentivirus, selected in puromycin, then drug-treated as indicated. Cell number (trypan blue, (b)) and apoptosis (PARP cleavage, (c)) were assessed on day $3(n=3$, mean $\pm \mathrm{SD}, * p<0.05$, $* * p<$ $0.01, * * * p<0.001$ ordinary one-way ANOVA, Tukey's multiple

\section{p53 induction of p21 mediates resistance to $\mathrm{B02}$ and TPT}

Drug resistance is a major reason for treatment failure, thus we investigated why WERI-RB1 cells resist B02 + TPT treatment. Resistance was recapitulated in vitro (Fig. 5a, b), providing a setting to test hypotheses. Unique to WERI-RB1,
B

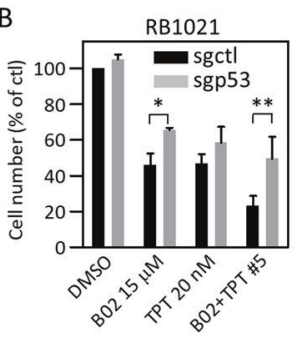

D

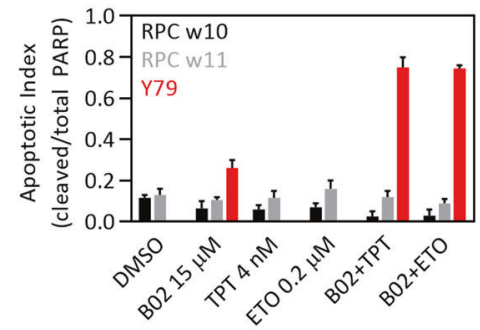

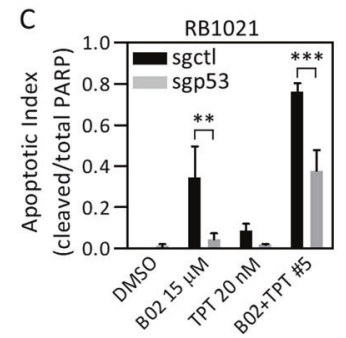
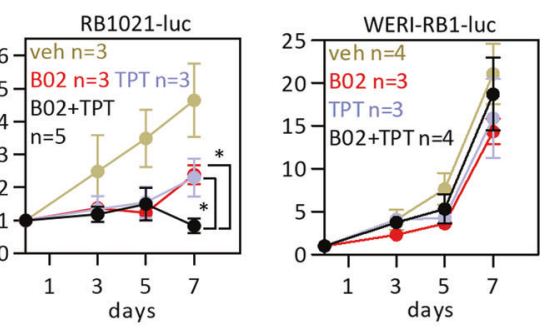

Fold

Amount $(\mu \mathrm{g})$ of single Reduction drug to achieve Fa (DRI)

\begin{tabular}{|c|c|c|c|c|}
\hline רחס & TDT & רחי & TIT & $C$ \\
\hline & & & & \\
\hline 34 & 0.013 & 3.4 & 10 & 0.39 \\
\hline 16 & 0.24 & 5.4 & 244 & 0.19 \\
\hline 0.11 & $3 \times 10^{-6}$ & 0.04 & 0.04 & 53 \\
\hline
\end{tabular}

comparisons test; representative PARP western in Fig. S16A). d RPC or Y79 tumor cells were treated with the indicated single drugs or combo \#5 from (a), and apoptosis quantified on day 3 (PARP cleavage, $n=2$, mean \pm range; representative PARP western in Fig. S16G). e Timeline to assess IVT B02 and/or TPT in three orthotopic RB xenograft models. f Based on day 7 data from Fig. S17A, B and plotted as dose response in Fig. S17C, subEC50 doses of B02 and TPT were selected (indicated in columns 2 and 3 of the table below the growth curves) and tested alone or together on the three indicated xenograft models (mean $\pm \mathrm{SD}, * p<0.05$ two-way ANOVA, Sidak's multiple comparisons test). DRI and CI are also indicated on the table. g Representative images of the radiance signals for the three RB tumors treated as indicated after 7 days are shown.

B02 alone or with TPT caused autophagy-like effects, including vacuolization and flattening (Fig. S18A). However, the autophagosome marker LC3-II was not induced, and two autophagy inhibitors did not affect LC3-II nor sensitize cells to B02 and/or TPT (Fig. S18B-D). Vacuoles in B02-treated cells lacked mitochondria, arguing against the idea that mitophagy inhibits the intrinsic apoptotic cascade (Fig. S18E). 
A
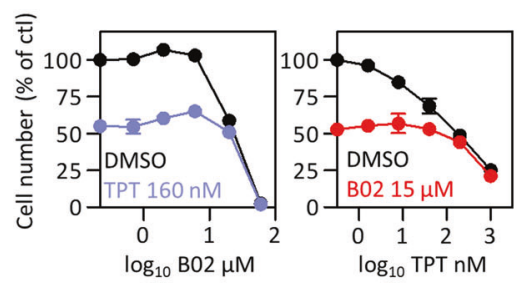

C
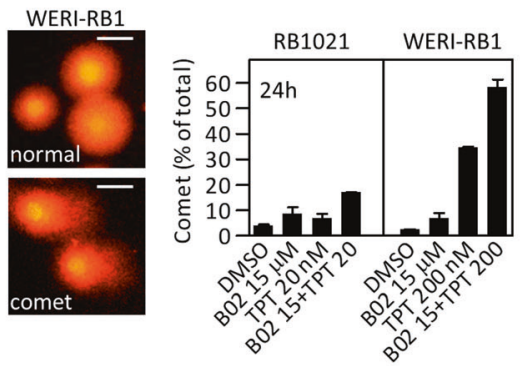

key for $E$ and $K$ DMSO

B02 $15 \mu \mathrm{M}$ TPT $200 \mathrm{nM}$ B02+TPT \#9

$\mathrm{H}$
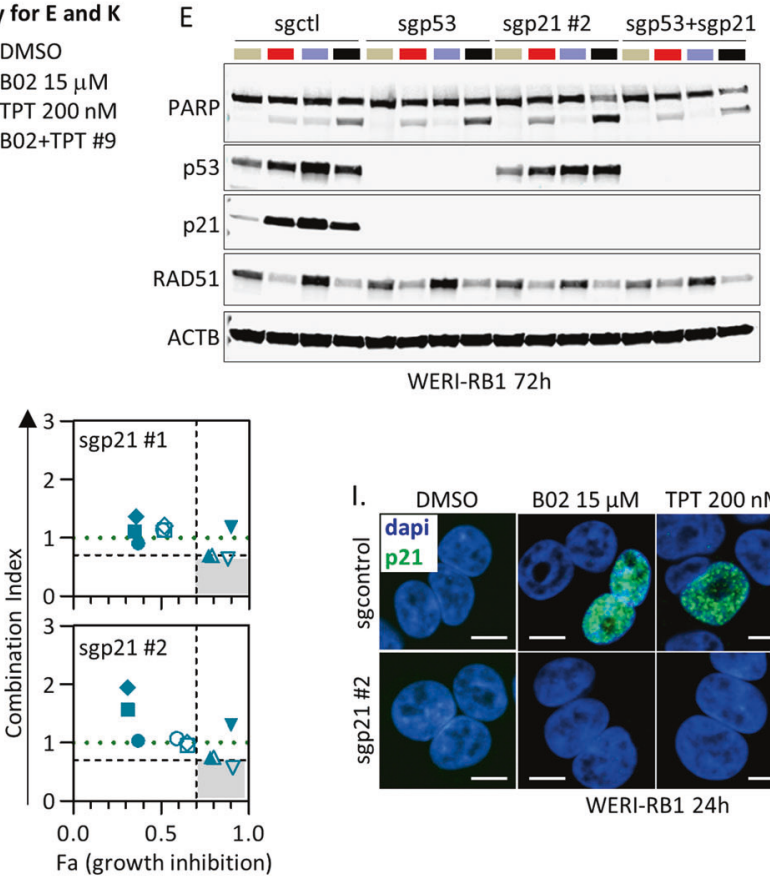

K

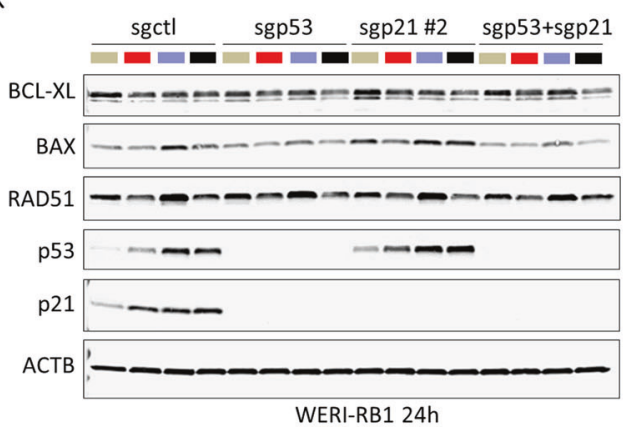

B

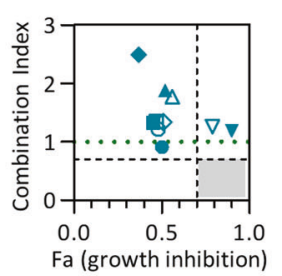

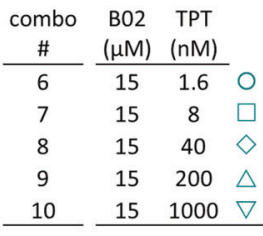

D
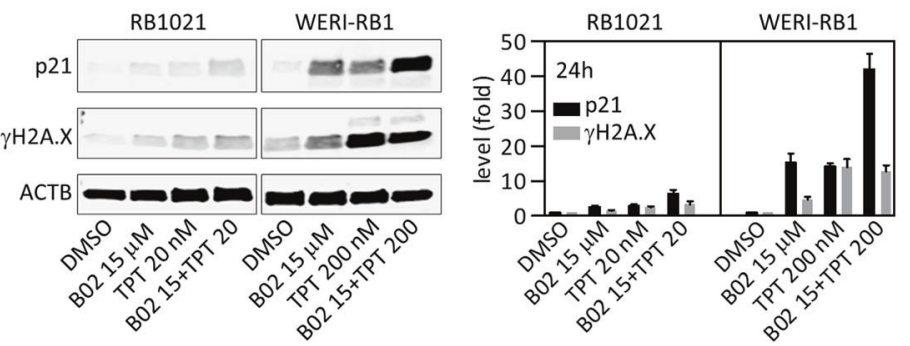

$\mathrm{F}$
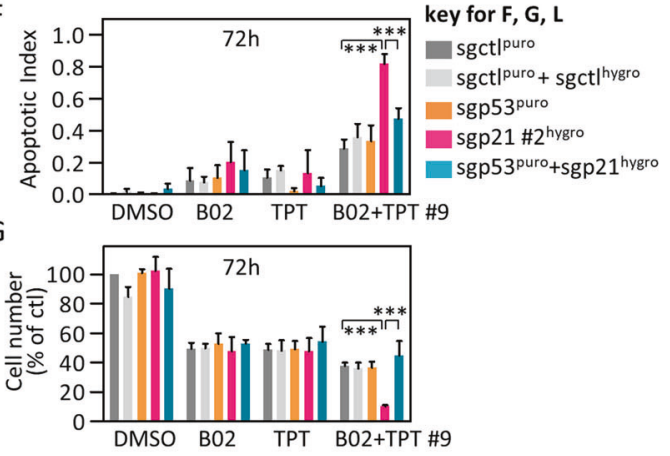

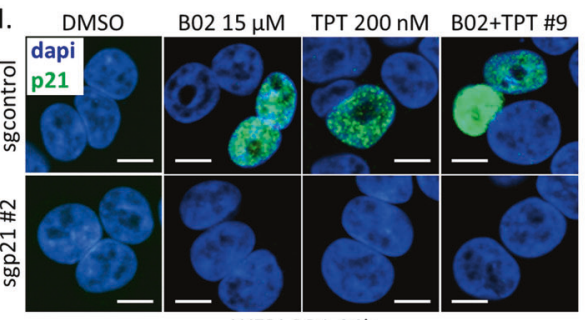

WERI-RB1 24h

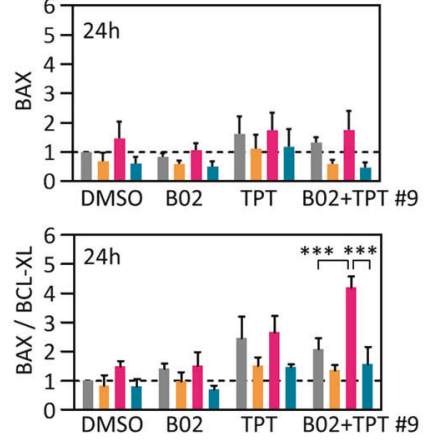

J $\quad G 1 \quad S(E d U+) G 2 / M$

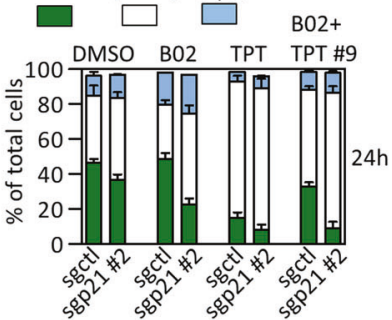

M

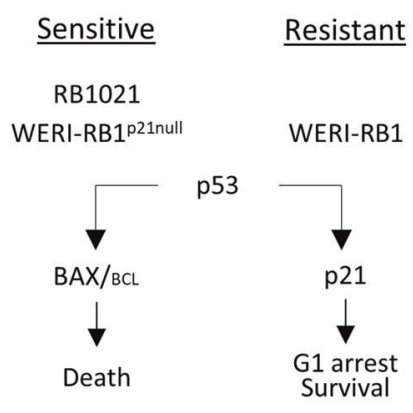

Comet assays and $\gamma \mathrm{H} 2 \mathrm{~A} . \mathrm{X}$ westerns revealed that B02 + TPT increases DNA damage vs. single drug, but unexpectedly, the combo induced greater damage in resistant WERI-RB1 vs. sensitive RB1021 cells (Fig. 5c, d). The p53 target, p21 can arrest cells to permit DNA repair [58], and indeed, $24 \mathrm{~h}$ post treatment it was induced to 
Fig. 5 The p53-p21 axis underpins resistance to B02 and TPT. a WERI-RB1 cells were treated with increasing concentrations of B02 (left) or TPT (right) together with vehicle (black) or EC50 doses of TPT (blue) or B02 (red) and cell number determined after 3 days (CellTiter-Glo, $n=2 \pm$ range). b The combos tested in (a) are summarized and $\mathrm{CI}$ vs. effect (Fa) plotted. Gray area and green line as in Fig. 4a. Sensitive RB1021 or resistant WERI-RB1 cells were treated $24 \mathrm{~h}$ with $\approx \mathrm{EC} 50 \mathrm{~B} 02$ and/or TPT and DNA double-stranded breaks assessed by alkaline comet assay (c) and $\gamma \mathrm{H} 2 \mathrm{~A} . \mathrm{X}$ westerns (d). Examples of propidium iodide-stained normal nucleoids, DNA comets, and normalized comet quantification ( $n=2 \pm$ range) are shown in (c). Scale bars are $10 \mu \mathrm{m}$. In (d), p21 and $\gamma \mathrm{H} 2 \mathrm{~A}$.X expression were also quantified ( $n=2 \pm$ range). e-g Deletion of $\mathrm{p} 53$, p21, or both with CRISPR/Cas9 sgRNA lentiviruses was performed in WERI-RB1, and sensitivity to B02, TPT, and B02 + TPT combo \#9 (from (b)) was assessed after 3 days by tracking apoptosis (representative blot in (e), quantified in (f)), and cell number $(\mathbf{g})(n=3$, mean $\pm \mathrm{SD}, * * * p<0.001$ ordinary one-way ANOVA, Tukey's multiple comparisons test). p53, p21, RAD51 levels were also assessed in (e). Additional westerns are shown in Fig. S19H. h B02/TPT synergy assay in p21-deleted cells, run as for parental cells in (a, b). The related growth inhibition curves for the two sgp21 tested are shown in Fig. S19G. i, j WT or p21-null WERI-RB1 cells were treated $24 \mathrm{~h}$ with single drugs or combo \#9 (from (b)) and p21 localization assessed by immunostaining (i), or cell cycle phase defined by EdU and fxcycle staining followed by flow cytometry (j) $(n=2$, mean \pm range). Representative flow plots are shown in Fig. S19I. Scale bars are $10 \mu \mathrm{m}$. $\mathbf{k}$ The experiment in $(\mathbf{e}-\mathbf{g})$ was repeated but protein levels were assessed at $24 \mathrm{~h}$. I BAX and BCL$\mathrm{XL}$ western blots in $(\mathbf{k})$ were quantified, normalized to actin and DMSO, then levels were plotted as indicated $(n=3$, mean $\pm \mathrm{SD}$, *** $p$ $<0.001$ ordinary one-way ANOVA, Tukey's multiple comparisons test). $\mathbf{m}$ Schematic summarizing the distinct p53 response to B02/TPT in sensitive (left) or resistant (right) contexts.

higher levels in WERI-RB1 cells relative to RB1021 with either drug alone and maximally with the combo (Fig. 5d). By $72 \mathrm{~h}$ p21 was lower in combo vs. single drug-treated cells, reflecting greater apoptosis in the former (Fig. 5e). CRISPR-mediated p21-deletion increased apoptosis and reduced cell number specifically in combo-treated WERIRB1 cells, thus drug resistance is p21-dependent (Fig. 5e-g, Fig. S19A-F). Dose-response experiments identified drug concentrations that synergistically killed p21-deficient WERI-RB1 cells (Fig. 5h, Fig. S19G), contrasting no synergy in parental cells (Fig. 5a, b). Cytoplasmic p21 inhibits caspase 3 [59], but the protein was nuclear (Fig. 5i). RAD51 levels, which were reduced by B02, were unaffected by p21 loss (Fig. 5e), suggesting another mechanism of resistance. In WT cells, TPT caused S-phase arrest, but adding B02 increased G1 cells to levels approaching vehicle or B02-only treated cells, thus p21 may protect combotreated cells through G1 arrest (Fig. 5j, Fig. S19I). Indeed, deleting p21 dramatically increased the fraction of combotreated cells in S-phase (Fig. 5j, Fig. S19I). Thus, while the combo induces greater DNA damage in drug-resistant cells, the counterintuitive reduction in death is due to more robust p21 induction, G1 arrest and protection of a subset of cells.

\section{Re-engaging the p53-BAX axis to kill B02/TPT- resistant cells}

The p53-BAX axis underpins B02 + TPT synergy in RB1021 cells (Fig. 4b, c, Fig. S16A). p21 is also a p53 target, thus conceivably p53 protects WERI-RB1 cells because p53-p21 predominates over p53-BAX signaling. Indeed, $24 \mathrm{~h}$ after B02 + TPT treatment, the BAX/BCL2 ratio was markedly induced in p21-deleted WERI-RB1 compared to resistant parental cells (Fig. 5k, 1). p53 was essential for the induction of p21 in WT WERI-RB1 cells (Fig. 5k, 1). In line with a context-dependent role, deleting p53 alone in WERI-RB1 cells did not alter drug responsiveness because neither p21 nor BAX/BCL-XL was induced (Fig. $5 \mathrm{e}-\mathrm{g}$ ). Thus, p53 promotes or antagonizes B02 + TPT synergy in RB cells depending on whether the BAX or p21 pathways predominate, and removing p21 restores BAX dominance in resistant cells (Fig. 5m).

\section{Synergism with Navitoclax overcomes resistance to B02 + TPT}

Next, we pursued a pharmaceutical strategy to bypass resistance to B02 + TPT. Our data reveal that, in all contexts, synergy engages proapoptotic BCL family proteins. Thus, we tested whether combining an inhibitor of antiapoptotic BCL proteins with B02 + TPT might overcome resistance. In addition, B02 or TPT alone activate p53 (Fig S16A, Fig. 5e, k), thus pairing either with a BCL inhibitor should also engage the BAX axis and drive synergy. Navitoclax (ABT-263) is an oral, bioavailable small molecule inhibitor of BCL2, BCL2L1, and less potently, BCL2L2 (BCL-w) [60]. We ran dose-response curves for Navitoclax, TPT, or B02 alone or in combination with fixed subEC50 doses of one (two-drug combos) or the other two drugs (three-drug combos). B02 + TPT combos had no effect, confirming resistance, but multiple TPT + $\mathrm{Nav}$ and $\mathrm{B} 02+\mathrm{Nav}$ dual combos synergistically killed WERI-RB1 cells (Fig. 6a-c). Triple combos were even more effective in all three dose-response curves (Fig. 6a, b). To quantify triple drug synergy we considered a drug pair as single entity, thus the pair is kept constant and the third drug is varied [53, 61], and indeed many triple drug combos were synergistic relative to two drugs (Fig. 6d). Finally, we showed that either B02 + Nav or TPT + Nav dual therapies were also effective in RB1021 cells, that triple therapy further enhanced apoptosis in both RB lines, and that p53 was essential for this effect with all these drug combinations (Fig. 6e). Each therapy induced p21 in WERI-RB1 but not RB1021 cells, indicating that Navitoclax overrides this defense (Fig. 6e). Navitoclax is thus a new pharmacological agent for RB that synergizes with TPT and/or B02 to bypass p53-induced cell cycle arrest and resistance, and instead 
A

WERI-RB1 24h

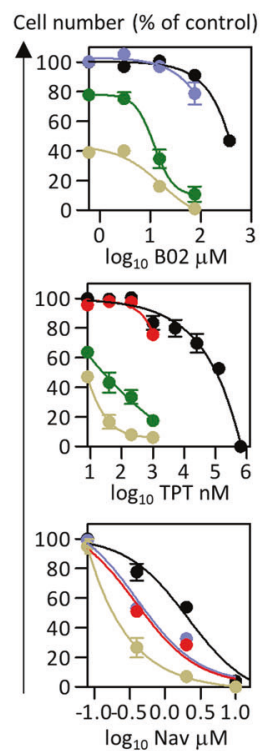

Apoptotic Index
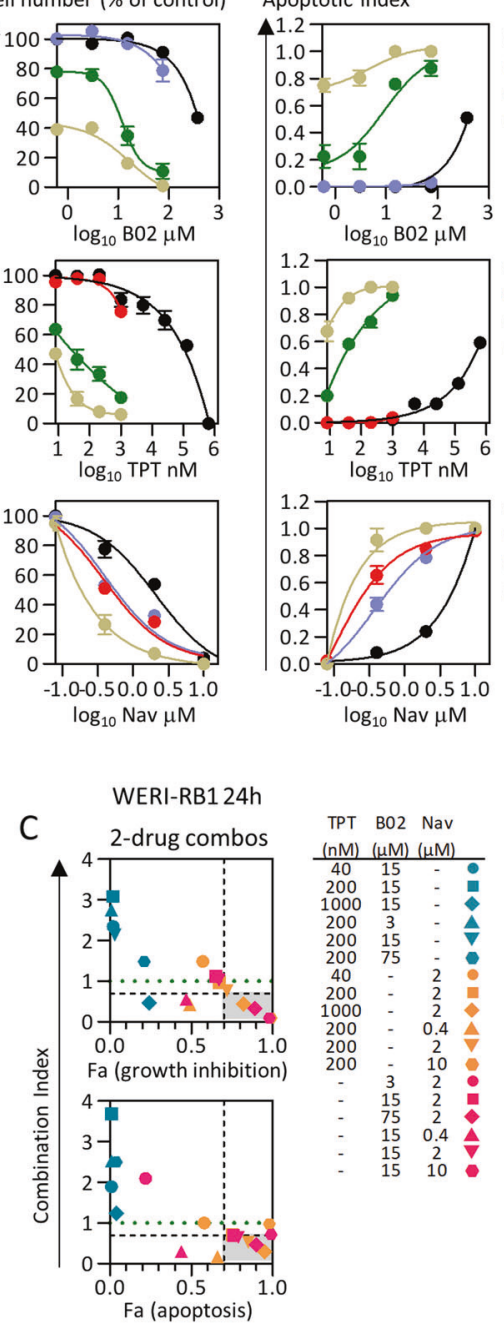

E

\begin{tabular}{|l|}
\hline sgctl \\
sgp53 \\
1. DMSO \\
2. $15 \mu \mathrm{M}$ B02 \\
3. $20 \mathrm{nM}$ TPT (RB1021) \\
3. $200 \mathrm{nM}$ TPT (WERI-RB1) \\
4. $2 \mu \mathrm{M} \mathrm{Nav}$ \\
5. B02 + TPT \\
6. B02 + Nav \\
7. TPT + Nav \\
8. B02 +TPT + Nav \\
\hline
\end{tabular}

TPT B02 Nav

$\frac{(\mathrm{nM})(\mu \mathrm{M})(\mu \mathrm{M})}{40 \quad 15}$

$\begin{array}{ccc}40 & 15 \div- \\ 200 & 15=0 \\ 1000 & 15=\end{array}$

2003

$200 \quad 15$

400
1000

200
1000
200
200

- $10=$

$\begin{array}{cc}3 & 2 \\ 15 & 2\end{array}$

$\begin{array}{ll}15 & 2 \\ 75 & 2\end{array}$

$\begin{array}{lcl}15 & 0.4 & \mathbf{A} \\ 15 & 2\end{array}$

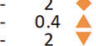

B
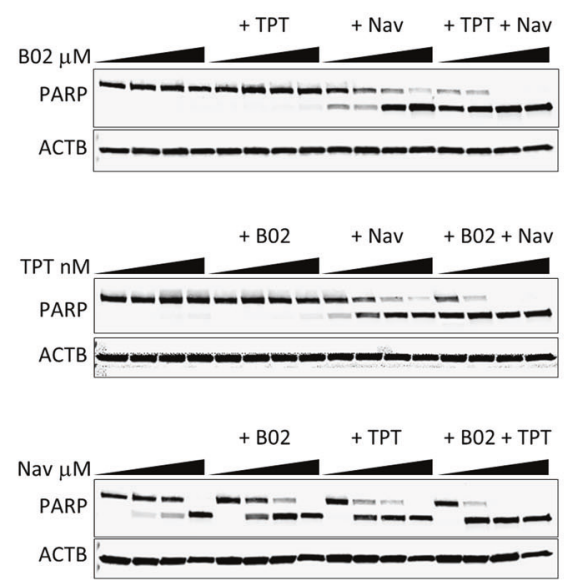

- DMSO

- TPT (200 nM)

- Nav $(2 \mu \mathrm{M})$

- TPT + Nav

DMSO

B02 $(15 \mu \mathrm{M})$

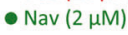

\section{- DMSO}

- TPT $(200 \mathrm{nM})$

- B02 $(15 \mu \mathrm{M})$

- TPT + B02

D WERI-RB124h

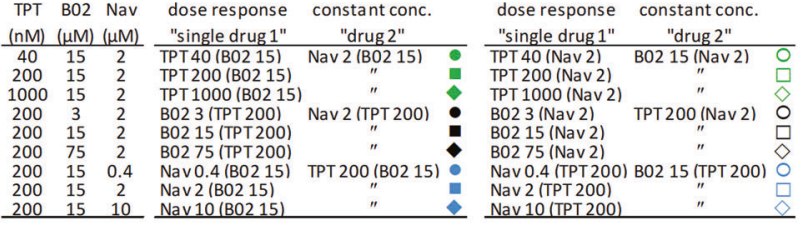

Triple drug synergy (vs two-drugs)

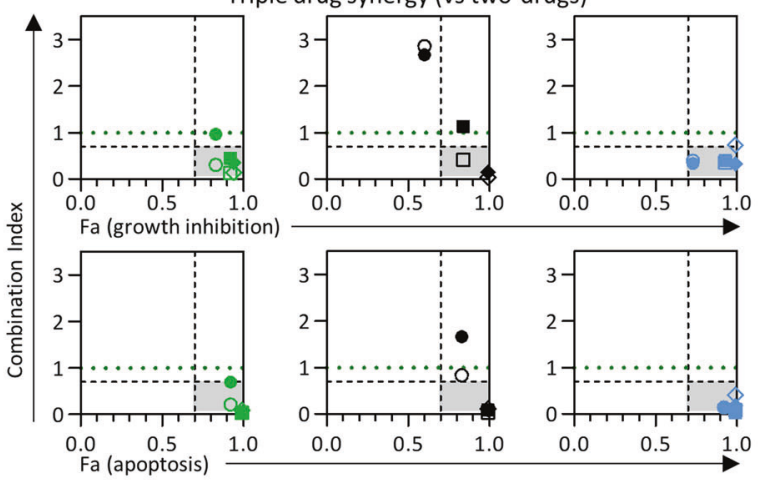

WERI-RB124h

RB102124h
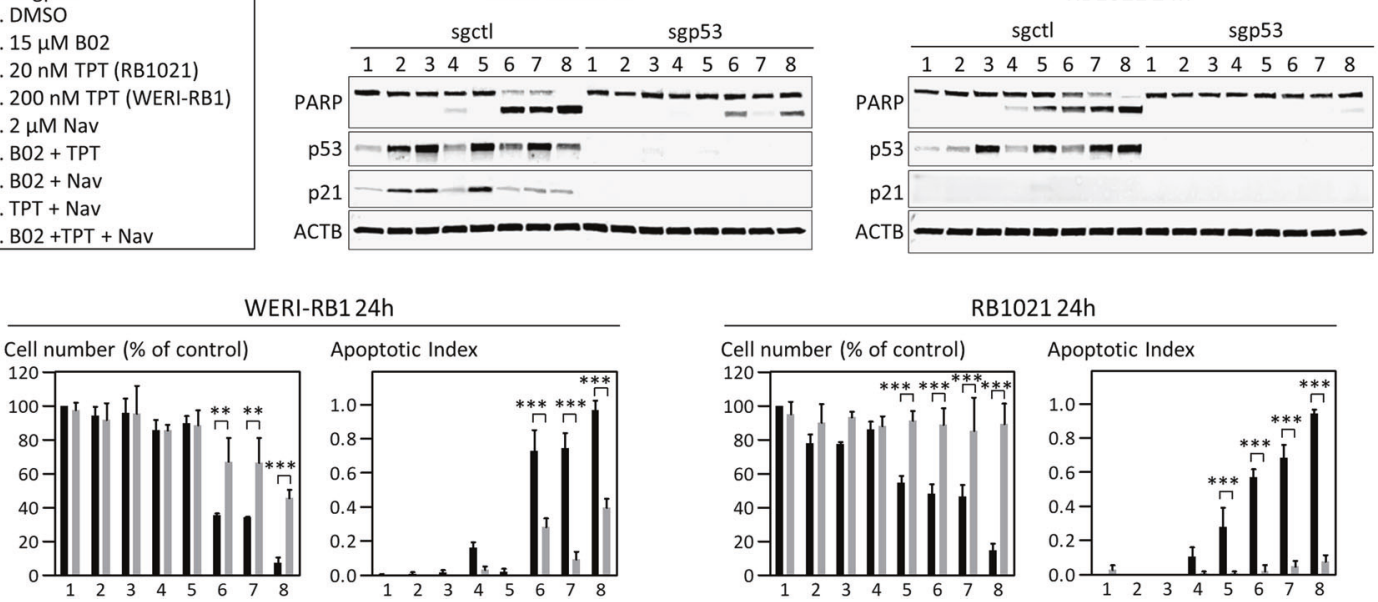
Fig. 6 Synergistic drug combinations to kill B02/TPT-resistant RB. a WERI-RB1 were treated with increasing concentrations of B02 (top), TPT (middle), or Navitoclax (Nav, bottom), and with vehicle (black), or with EC50 doses of TPT (blue, two-drug combos), B02 (red, two-drug combos), or Nav (green, two-drug combos), or pairs of EC50 drugs (brown, three-drug combos), and cell number (a, left graphs) and apoptosis (a, right graphs) defined after 3 days ( $n=2 \pm$ range). b Representative PARP western used to quantify apoptosis in (a). The two-drug (c) or three-drug (d) combos from (a) are summarized in the tables, and CI vs. effect (Fa) graphed (gray areas and green lines as in Fig. 4a). e The indicated RB cell lines were transduced with control or p53-targeting sgRNA lentivirus, selected in puromycin, then $7 \times 10^{5}$ cells were seeded and treated as indicated. At $24 \mathrm{~h}$, cell number (trypan blue) and apoptosis (PARP) were quantified $(n=3$, mean $\pm \mathrm{SD}, \quad * * p<0.01, \quad * * * p<0.001$ ordinary one-way ANOVA, Tukey's multiple comparisons test). Representative PARP, p53, and p21 western blots are shown.

engage p53-driven apoptosis. The triple therapy may be generally applicable to most RB tumors irrespective of the dominance of the p53-BAX vs. p53-p21 axes.

\section{Discussion}

Coupling dynamic network analysis with functional genomics, we uncovered new synergistic therapies for a pediatric cancer. Network analysis prioritized 61 candidates for an in vivo shRNA screen in orthotopic xenografts of two $R B l^{\text {null }}$ lines, which were compared with 86 controls. Validation screens were run with 21 primary hits and 34 controls in three $R B 1^{\text {null }}$ lines and an $R B 1^{w t} ; M Y C N^{a m p}$ line, yielding 15 genes that were positive in both screens. These high value hits included known RB drivers (e.g., SKP2, MYCN, ID2), validating the screen, but also the RAD51/BRCA1 complex and various interacting partners. BRCA1 is a well-known tumor suppressor, but also has oncogenic roles with RAD51 through its DNA-repair function [62, 63]. We excluded other BRCA1 functions, but implicated BRCA1 and RAD51 in maintaining DNA integrity in RB cells. RB cells, but not normal fetal progenitors, underwent apoptosis upon RAD51 depletion. While cultured RPC are not a normal counterpart to $\mathrm{RB}$ cells, they provide an example of a proliferating $R B I^{w t}$ cell type that is insensitive to combined RAD51- and topoisomerase-inhibitor therapies. To test the role of pRB loss in sensitizing to this combination it may be of interest to define B02 + TPT effects on $R B I^{w t} ; M Y C N^{a m p}$ RB cells. The selectivity for RAD51 loss in $R B 1^{\text {null }} \mathrm{RB}$ cells vs. RPC was reproduced with B02, a small molecule RAD51 inhibitor. Furthermore, B02 synergized with TPT or ETO, front line drugs in RB treatment, and these combinations also did not affect human RPC survival.

These positive results led us to test B02 + TPT in vivo. We observed synergistic efficacy in Y79 and RB1021, but not WERI-RB1 xenografts. These assays were performed over 7 days and with a vitreal model of disease, thus longerterm studies should be carried out prior to clinical tests, and additional models are required to examine effects on retinal disease. Mechanistically, B02 + TPT engaged the p53-BAX apoptotic axis in sensitive RB cells, but a protective p53-p21 G1-arrest pathway in drug-resistant cells. Deleting p21 switched the p53 response to engage BAX, driving apoptosis. That result suggested that $\mathrm{BCL}$ family inhibitors might bypass resistance, and indeed the BCL2/ BCL-XL (BCL2L1)/BCL-W (BCL2L2) inhibitor Navitoclax synergized with either B02 or TPT, and the triple therapy was even more potent. This approach was effective with either B02 + TPT-sensitive RB1021 or B02 + TPTresistant WERI-RB1 cells. Thus, Navitoclax warrants further testing as a new strategy to enhance TPT-killing of RB tumors, and RAD51 inhibitors could further heighten efficacy.

The synergy observed with TPT, B02, and/or Navitoclax in RB suggests these combinations may be effective in other p5 $3^{\mathrm{wt}} R B 1$-deficient cancers. Whether they could be exploited in $R B 1 / p 53$-deficient cancers, such as small cell lung cancer (SCLC) or triple negative breast cancer (TNBC) is unclear. Recently, synergistic effects have been described in TNBC using CDC25 inhibitors plus WEE1 or PI3K inhibitors [64], as well as a synthetic lethal interaction between RBI-deficiency and Aurora Kinase A or B inhibitors in SCLC or TNBC [65, 66]. It will be interesting to compare the efficacy of these approaches with the TPT/B02/ Navitoclax strategies outlined here in RB. A recent study found upregulation of BCL2 and WEE1 kinase in small cell neuroendocrine (SCN) cancers, and combining Navitoclax and the WEE1 inhibitor AZ-1775 was synergistic against SCN prostate cancer patient-derived xenografts [67]. Navitoclax was also partially successful in a phase II clinical trial for SCLC, with $23 \%$ of patients (9/39) showing stable disease and one patient with a partial durable response [68]. A drawback was the high rate of thrombocytopenia, which would not be a risk with localized IVT injection for RB. Follow-up work revealed an unusually high level of BCL2 in many SCLC tumors relative to most other solid cancers, which correlated with sensitivity to the BCL2-selective inhibitor, Venetoclax [69]. Thus, BCL inhibitors, especially in combination with synergistic drugs, may enhance therapeutic outcome in highly lethal RBdeficient SCN cancers.

Although dual combinations were potent, the most effective mix was the triple combination of TPT, B02, and Navitoclax. B02 has not been tested in the clinic, and as a single agent high $\mu \mathrm{M}$ concentrations are required, limiting its usefulness in vivo. Of note, CYT-0851 is another RAD51 inhibitor that was recently approved for a phase $1 / 2$ clinical study in various cancers (NCT03997968) after showing promise in preclinical models [70], thus may be a 
potential valuable alternative. We observed potent in vitro synergy in triple therapy with $15 \mu \mathrm{M} \mathrm{B} 02$, and although we did not test this combination in vivo, B02 enhanced the response to TPT in preclinical trials (Fig. 4). Intravenous pharmacokinetics and toxicology cannot predict IVT or intra-arterial drug behavior, so preclinical studies are needed to assess clearance and toxicity in the eye with these modalities. Since TPT is currently used to treat RB, and Navitoclax has been approved for use in humans, the first priority will be to further test this combination in preclinical models and, if effective and safe, to bring this combination to the clinic.

\section{Materials and methods}

\section{RNA and microarrays}

Human fetal retinas were obtained from the Morgentaler Clinic in Toronto with approval from the Research Ethics Board (REB \#13-0132-E) of Mount Sinai Hospital in Toronto, Canada, and consent from patients. Gestational age was estimated by a combination of clinic intakes, ultrasound, crown-rump, and fetal foot length measurements where possible [71, 72]. Eye samples collected were held on ice for up to $6 \mathrm{~h}$ in the retina culture medium (IMDM with 10\% FBS and 1× Antibiotic-Antimycotic (Life Technologies, Toronto, ON, Canada)). RB samples were obtained at the Vision Research Foundation, Sankara Nethralaya Chennai, India, with the approval from the institutional research and ethics board, and consent from patients. RNA quality was determined using Agilent 2100 Bioanalyzer and only medium-high quality samples were processed. RNA was reverse transcribed and hybridized to Illumina Human V6 beadchips (v2, San Diego, CA, USA). Murine RNA samples were collected using RNeasy Mini kit (Qiagen, Venlo, The Netherlands) from retina of mixed C57BL/6 $\times$ 129SvJ backgrounds. RNA quality was determined using Agilent 2100 Bioanalyzer (TCAG, Toronto, ON, Canada). Only samples with an RNA Integrity score of $\geq 7$ were used. Reverse transcription and hybridization were performed by TCAG, Toronto. Samples were hybridized to Illumina Mouse WG6 beadchips (v1.1, San Diego, CA, USA). Probe intensity scores were processed using RMA background correction and $\log 2$ transformation.

\section{DyNeMo}

We focussed on human/mouse orthologues present in the OPHID network. From 24,582 human and 13,544 mouse geneids we identified 10,633 homologous geneids. We mapped the geneids to the OPHID ppi skeleton (formed by the edges with the highest betweenness centralities of the origin network, which is believed to preserve the modular structure of biological network while greatly simplifying the complexity of the highly entangled origin network), of which 4480 remained after removing singletons. We first ran DyNeMo on 21 human RB transcriptomes vs. 12 normal human fetal retina $(4 \times$ week $12,4 \times$ week $15,4 \times$ week 18) transcriptomes. Three DyNeMo runs were performed, with each run using a randomly selected two-thirds of the samples (14 tumors, 8 normals). We selected hubs that were significant in all three runs by nonparametric $p$ values, generated by comparing the mean absolute difference of the Pearson correlation coefficient in the tumor/normal samples versus 1000 randomly generated samples. To obtain evolutionarily conserved hubs, we repeated DyNeMo with transcriptome data from 14 normal murine retinal samples (3 E15.5, 3 P0, 4 P8, 4 adult/1mo) and 23 murine RB samples, harvested when the affected adult eye was full of

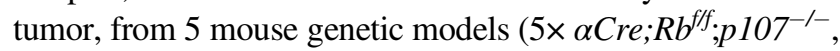

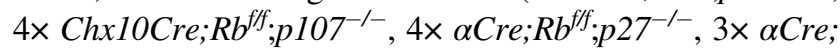
$R b^{f f f} ; p 27^{C K-/ C K-}, 3 \times \alpha C r e ; R b^{f f f} ; p 130^{-/-}, 4 \times$ TAg-RB). All animal experiments were conducted with ethical approval from the animal care committees of each research institute (University Health Network for all models, except $\alpha C r e$; $R b^{f f} ; p 130^{-/-}$, in which case mRNA was a gift of David Macpherson at the Fred Hutchinson Cancer Research Centre, Seattle, USA; TAg-RB samples were a gift from Brenda Gallie). The models have been described previously [73-77].

\section{RNAi screens and validation}

A custom pool of bar-coded shRNAs in the pLKO.1 lentiviral backbone targeting DyNeMo candidate hubs (detailed in Table S1) was first cherry picked from The RNAi Consortium (TRC) library, then pooled lentiviruses were produced according to standard protocols (https://portals.broadinstitute. org/). In the primary screen, WERI-RB1 or Y79 cells were transduced at $\mathrm{MOI}=0.2$ and selected in puromycin. T0 genomic DNA (gDNA) was isolated from some of the cells, and the remainder was orthotopically transplanted into NODSCID eyes (250,000 per eye). Six tumors were grown per cell line. After large tumors formed (4-6 weeks) deep sequencing was performed and revealed 0.1-27,000 normalized reads per virus, but $\sim 95 \%$ shRNAs yielded $\geq 10$ normalized reads (Fig. S2) [78]. Outlier exclusion was performed prior enrichment analysis. Briefly, normalized reads were $\log$ transformed for data linearization then robust $Z$-scores for each shRNA in every sample were obtained, allowing outlier identification (Irobust $Z \mid>5$ ) and exclusion of $\leq 2 / 6$ tumors per shRNA for each cell line, leaving $\geq 4$ tumor replicates per shRNA for enrichment/depletion analysis. Next, averaged tumor/T0 counts for each shRNA minus outliers were obtained and $\log$ transformed. Fold-change was then 
converted into $Z$-scores after confirming that the distribution of $\log$ tumor/T0 dataset followed a Gaussian pattern (Fig. S2D, Table S1). For the second screen with four RB cell lines, hit shRNAs from screen 1 as well as an equal number of nonscoring shRNAs were selected (detailed in Table S1). The same procedure was followed to generate pooled viruses, transduce cells, and collect T0 and tumor gDNAs. The generated data were not Gaussian, reflecting the reduced $\mathrm{n}$ and high hit fraction in the second screen, thus $Z$-scores could not be calculated, so a stringent cutoff of fivefold reduced tumor growth (log ratio -0.69 ) and $\geq 2$ scoring shRNAs was used to establish robust hits (shaded area in Fig. 2d). Hits were further classified as high, medium, or low quality if they scored in $\geq 3$, 2, or 1 of the 4 cell lines, respectively (Fig. 1f, Table S1).

For validation, Y79 cells were transduced with shRNAs viruses scoring in both screens. Knockdown efficiency was assessed by western blot, and cell number by trypan blue. The following shRNAs from TRC shRNA library were used: luciferase (negative control) TRCN0000072256; BRCA1 \#1 TRCN0000244985, BRCA1 \#2 TRCN0000244986; RAD51 \#1 TRCN0000018876, RAD51 \#2 TRCN0000329688; SKP2 \#1 TRCN0000007534, SKP2 \#2 TRCN0000315078; PABPC1 \#1 TRCN0000074639, PABPC1 \#2 TRCN0000074641; MSI \#1 TRCN0000062811, MSI \#2 TRCN0000062812.

\section{Cell culture}

Dissociated RPC were cultured in Iscove's modified Dulbecco's medium supplemented with $10 \%$ fetal bovine serum (FBS), 1× 2-mercaptoethanol (Gibco, ThermoFisher Scientific, Burlington, ON, Canada), $10 \mu \mathrm{g} / \mathrm{ml}$ plasmocin, and $1 \times$ anti-anti (Gibco). The low passage RB cells lines RB3823, RB3535S, RB4036, RB4063, RB3935 were grown in T25 flasks in Iscove's medium supplemented with 10\% FBS, antibiotics, 0.0004\% (v/v) 2-mercaptoethanol, and $10 \mu \mathrm{g} / \mathrm{L}$ insulin as previously described [79]. We cultured RB1021 cells (gift from Brenda Gallie) in the same conditions as the low passage RB lines, WERI-RB1 and Y79 cells were cultured in RPMI1640, A549 cells in DMEM, H661 in RPMI1640, PFSK-1 (gift from Dr. Annie Huang) in DMEM with $1 \times$ MEM NEAA (Gibco), and all were supplemented with $10 \%$ FBS and antibiotics. Cell lines were mycoplasma free. We performed STR analysis on four of the RB lines, two of which have STR profiles (Y79, WERI-RB1) and two of which do not (RB3823, RB1021), so the latter will be useful to others in the future; the information is provided in Table S2. The STR profiles for Y79 and WERI-RB1 were 97 and 84\% matches and designated as "derived from a common ancestor" by the test lab (The Centre for Applied Genomics, Hospital for Sick Children, Toronto, Canada), consistent with modest drift from the original source. For the other early passage cell lines mentioned in the westerns for Fig. 1g, those lysates were a gift from Dr. Brenda Gallie (Hospital for Sick Children, Toronto). Dr. Gallie was routinely generating and genotyping tumor lines at that time and is heavily involved in genotyping patients/tumors, thus we relied on their considerable expertise to assure authenticity.

\section{Western blotting}

Cells were lysed for $1 \mathrm{~h}$ on ice in RIPA buffer (Santa Cruz, Mississauga, ON, Canada) supplemented with protease inhibitor cocktail, sodium orthovanadate and PMSF. Lysates were run on 4-20\% SDS-PAGE gradient gels, transferred to nitrocellulose, and analyzed by Li-Cor system (LI-COR Biosciences, Lincoln, NE, USA) with antibodies listed in Table S3.

\section{Cell growth assays}

2D culture; 96-well format: RB cells were seeded at 10,000 cells/100 $\mu$ l. MDA-MB-231 cells were seeded at 3000 cells/ $100 \mu \mathrm{l}$. Drugs were prepared by serial dilutions at $6 \times$ concentrations and $20 \mu \mathrm{l}$ were added to the cells. Cell viability was assessed at different timepoints with CellTiter-Glo Reagent (Promega, Madison, WI, USA), and luminescence quantified with a plate reader (EnVision, PerkinElmer, Woodbridge, ON, Canada). Six-well format: RB cells and RPC were seeded at 700,000 and 300,000 cells $/ 2 \mathrm{ml}$, respectively. The next day (day 0 of the assay), drugs, siRNAs, or doxycyclin were prepared at $5 \times$ and $0.5 \mathrm{ml}$ added to cells. Cell counts were performed with trypan blue.

3D culture; 96-well format: each well was first plated with $50 \mu \mathrm{l}$ of medium containing $0.6 \%$ agar, then Y79 cells were seeded at 600 cells $/ 60 \mu \mathrm{l}$ medium containing $0.35 \%$ agar. Drugs were prepared at $2.8 \times$ and $60 \mu$ added on top of the cell layer. After 6 days, colonies were assessed with Alamarblue Cell Viability Reagent (ThermoFisher, Burlington, ON, Canada), as described [80], and absorbance quantified with a plate reader as above.

\section{siRNA-mediated gene silencing}

Cells were seeded in six-well plates in medium without antibiotics. RB and PFSK-1 cells were seeded at 700,000 cells/2 ml, and A549, H661 cells at 200,000 cells/2 ml. siRNA mixes with DharmaFECT 1 Transfection Reagent (Horizon Discovery) were prepared as per the manufacturer's instructions at a final concentration of $50 \mathrm{nM}$ unless specified otherwise. Mixes were added at day 0 (and day 3 if assay $>3 \mathrm{~d}$, and cells were passed if $>80 \%$ confluent). siRNAs were from Qiagen: negative control SI03650318; BRCA1 SI02654575, SI02664368; RAD51 SI00045010, SI02629837; and Dharmacon siGenome: p53 D-003329-07. 


\section{Re-expressing RAD51 in siRAD51-treated cells}

RAD51 was re-expressed using a tetracycline-inducible system (Takara Bio, Mountain View, CA, USA). Briefly, RAD51 cDNA fused with Flag in C-terminal was cloned into the pLVX-TRE3G-mCherry vector and empty or RAD51 pLVX-TRE3G-mCherry lentiviruses as well as pLVX-Tet3G viruses were produced in 293T cells. Y79 cells were then sequentially transduced with the Tet3G virus, selected with $1 \mathrm{mg} / \mathrm{ml} \mathrm{G} 418$ for 7 days, then transduced with the second empty or RAD51 TRE3G-mCherry virus, and selected with $3 \mu \mathrm{g} / \mathrm{ml}$ puromycin for 7 days. RAD51 expression was induced with $2 \mu \mathrm{g} / \mathrm{ml}$ doxycyclin and cells were treated with a control or siRNA targeting the $3^{\prime}$ UTR region of RAD51 $24 \mathrm{~h}$ later.

\section{Cell fractionation}

Protein lysates from 500,000 cell equivalents were prepared with the subcellular protein fractionation kit for cultured cells (ThermoFisher, Burlington, ON, Canada). Total protein lysates were extracted in RIPA buffer (see "Western blot" section).

\section{Fluorescence microscopy}

\section{Cultured cells}

In six-well plates, coverslips were precoated with $50 \mu \mathrm{g} / \mathrm{ml}$ of poly-D-lysine (Sigma, Markham, ON, Canada) and $700,000 \mathrm{RB}$ cells and 300,000 dissociated RPC were seeded in $2 \mathrm{ml} /$ well overnight. Where applicable, cells were labeled with MitoTracker Deep Red FM (Cell Signaling, Whitby, ON, Canada) or EdU as per manufacturer's instructions. Cells were fixed with $4 \%$ paraformaldehyde for 10-15 min, permeabilized and blocked for $1 \mathrm{~h}$, then subjected to click chemistry and probed with primary antibodies overnight at $4{ }^{\circ} \mathrm{C}$ as in Table S3. After 2-3 washes, cells were probed with Alexa Fluor 488- or 568-conjugated secondary antibodies (ThermoFisher, Burlington, ON, Canada) and 4,6diamidino-2-phenylindole or propidium iodide (PI) for $90 \mathrm{~min}$. Coverslips were mounted using VectaShield (Vector Laboratories, Burlington, ON, Canada). High resolution confocal images were acquired with the Wave FX Spinning Disc Confocal microscope (Quorum Technologies, Puslinch, ON, Canada) and Volocity software.

\section{Cryosections}

Mice were i.p. injected with $10 \mathrm{mg} / \mathrm{kg}$ EdU for $1 \mathrm{~h}$ before sacrifice. Frozen eye sections were blocked and permeabilized in PBS with 5\% donkey serum and $0.1 \%$ Tween 20 for
$1 \mathrm{~h} 30$ at RT. Slides were incubated with the click reaction mix, and antibody staining performed as described [74, 81].

\section{Cell cycle, EdU/DNA staining}

Cells in six-well plates were labeled using the Click-iT EdU Alexa Fluor 647 Flow Cytometry Assay Kit then counterstained for DNA with FxCycle Violet dye (ThermoFisher, Burlington, ON, Canada). At least 10,000 single cells per sample were acquired using the Gallios flow cytometer (Beckman Coulter, Mississauga, ON, Canada) and analysed with Kaluza software.

\section{High throughput detection of DSBs in the cell cycle}

Y79 cells were processed as described in the Edu/DNA content staining section and after the click chemistry step the cells were probed with $\gamma \mathrm{H} 2 \mathrm{~A}$.X antibody in blocking/ permeabilization buffer overnight at $4^{\circ}$. The cells were then washed $2 \times$ in PBS with $0.1 \%$ saponin for $10 \mathrm{~min}$, probed with Alexa Fluor 488-conjugated secondary antibody at $1 /$ 500 dilution (ThermoFisher Scientific, Burlington, ON, Canada) for $90 \mathrm{~min}$ at RT rotating, washed $2 \times$, and stained with $1.5 \mu \mathrm{g} / \mathrm{ml} \mathrm{FxCycle}{ }^{\mathrm{TM}}$ Violet dye for $1 \mathrm{~h}$. At least 10,000 single cells per sample were acquired with the Amnis ImageStreamX Mark II Imaging Flow Cytometer to detect nuclear $\gamma \mathrm{H} 2 \mathrm{~A}$.X foci per cell in the different phases of the cell cycle. Samples were analyzed with the IDEAS v6.2 software.

\section{Annexin V and FxCycle Violet staining}

Live cells were washed with PBS and resuspended in $150 \mu \mathrm{l}$ staining buffer with alexa Fluor 488 annexin V (ThermoFisher, Burlington, ON, Canada) and $1.5 \mu \mathrm{g} / \mathrm{ml} \mathrm{FxCycle}$ Violet dye for $25 \mathrm{~min}$ at RT. At least 10,000 single cells per sample were acquired using flow cytometry as above.

\section{Mitochondrial oxidative stress}

Cells were labeled with Mitosox red mitochondrial superoxide indicator (ThermoFisher Scientific, Burlington, ON, Canada) as per manufacturer's instructions. Fluorescence intensity was quantified and analyzed by flow cytometry as above.

\section{Bright field images}

Hematoxylin and eosin (H\&E)-stained sections were captured with the Olympus BX61 microscope, and cultured cells were captured with the Zeiss Axio Vert.A1 microscope. 


\section{Compounds}

TPT hydrochloride (HPLC: 99\% purity, diluted in DMSO for in vitro assays and sterile PBS for in vivo assays), ETO (HPLC: 99\% purity, diluted in DMSO), Navitoclax (HPLC: 98\% purity, diluted in DMSO), PF-477736 (HPLC: 99\% purity, diluted in DMSO), MK-8776 (HPLC: $96.4 \%$ purity, diluted in DMSO), KU-55933 (HPLC: 99\% purity, diluted in DMSO), 3-methyl adenine (HPLC: $99 \%$ purity, diluted in sterile water), and wortmannin (HPLC: $99 \%$ purity, diluted in DMSO) were purchased from Selleckchem (Cedarlane, Burlington, ON, Canada). The RAD51 inhibitor B02 (HPLC: 98\% purity, diluted in DMSO for in vitro assays and sterile PBS for in vivo assays) was purchased from Calbiochem (Millipore, Etobicoke, ON, Canada), the CHK2 inhibitor II (HPLC: 98\% purity, diluted in DMSO) from Sigma (Markham, ON, Canada), and Trolox (HPLC: 97\% purity, diluted in DMSO) from Santa Cruz (Mississauga, ON, Canada).

\section{CRISPR-mediated gene knockout}

sgRNA sequences were cloned into the LentiCRISPR v2 vector (Addgene Plasmid \#52961), or for double knockout cells, sgControl or p21 sgRNA \#2 were cloned into a modified version where the puromycin resistance gene was replaced with hygromycin. Guide sequences were as follow: sgctl: CGCTTCCGCGGCCCGTTCAA; p21 sgRNA \#1: TCAGAACCCATGCGGCAGCA, p21 sgRNA \#2: GTC ACCGAGACACCACTGGA; p53 sgRNA: CCATTGTT CAATATCGTCCG. Briefly, we transduced WERI-RB1 cells with the indicated sgRNA viruses. Four days after transduction, cells were selected in $1 \mu \mathrm{g} / \mathrm{ml}$ puromycin for 5 days to generate stable knockouts. To generate double knockout cells, sgControl or p53 knockout cells were transduced with an sgControl (hygromycin) or sgp21 (hygromycin) virus. Four days after transduction, cells were selected in $50 \mu \mathrm{g} / \mathrm{ml}$ hygromycin B for an additional 6 days.

\section{Synergy determination}

CI and DRI were computed with CompuSyn software based on the effects on either growth inhibition or apoptosis. The effect-oriented $\mathrm{Fa}-\mathrm{CI}$ plot was used to represent the data because a normalized isobologram would be over-crowded: Fa (growth inhibition), or Fa on growth inhibition, represents the inhibitory effect of a combo on cell number, and is expressed as $1-\left(X_{\text {combo }} /\right.$ $\left.X_{\mathrm{ct}}\right)$, where $X$ is cell number, and therefore a value of 0 means no effect compared to control, whereas 1 equals complete growth inhibition; $\mathrm{Fa}$ (apoptosis) represents the apoptotic index, a value of 0 means no apoptosis, whereas 1 equals complete apoptosis [53]. We increased stringency to highlight the most potent combinations based on three criteria: (1) $\mathrm{Fa}>0.7$ in the combo, reflecting major enhancement; (2) $\mathrm{CI}<0.7$, reflecting synergy; and (3) Criteria \#1, 2, met whether we varied BO2, TPT, or ETO. Criteria (1) and (2) delineate a shaded area on the $\mathrm{Fa} / \mathrm{CI}$ plots representing the therapeutically relevant synergy. Synergy of three-drug combinations was performed as previously described [61].

\section{Comet assay}

Cells were processed for the alkaline comet assay (detection of DSBs) according to the manufacturer's instruction (Trevigen, Cedarlane, Burlington, ON, Canada). DNA comets were stained with $1.5 \mu \mathrm{g} / \mathrm{ml}$ PI for $1 \mathrm{~h}$ at RT in the dark. At least 300-400 nucleoids (normal or comet) per assay were quantified with the Wave FX Spinning Disc Confocal microscope (Quorum Technologies, Puslinch, ON, Canada)

\section{Efficacy in orthotopic xenograft}

Animal protocols were in accordance with local and national guidelines. IVT injections were performed under general anesthesia using isoflurane. Human RB cell lines stably expressing luciferase were prepared at 25,000 cells/ $\mu \mathrm{l}$ in sterile PBS with $10 \%$ matrigel (BD Bioscience, San Jose, CA, USA) and $5 \%$ trypan blue, then $2 \mu$ of the mixture were injected into the right vitreous of 3-4 weeks old male NOD-Scid mice. Uninjected left eye served as a negative control. After seven days, B02, Topotocan, a mix of B02+ TPT, or vehicle (PBS) was injected randomly in tumorbearing eyes at the indicated doses (three animals/dose unless specified otherwise). $\mathrm{Luc}^{+}$tumor cells were tracked live by i.p. injection of D-luciferin at $150 \mathrm{mg} / \mathrm{kg}$ for $10 \mathrm{~min}$ and radiance total flux (photons/second) tracked with the Xenogen IVIS Imaging System 100 (PerkinElmer, Woodbridge, ON, Canada). At each timepoint, biological replicates were normalized to day 0, averaged, and plotted. Data acquisition was blinded.

\section{Eye toxicity}

B02 was injected in the vitreous of anesthetized 3-4 weeks old Nod-Scid mice at $0.3,3,10,30 \mu \mathrm{g}$, and 3 days later the mice were sacrificed, the eyes enucleated and incubated in Davidson's fixative overnight at $4{ }^{\circ} \mathrm{C}$ on a shaker. Ethanol dehydration and paraffinization of the tissue were done in a tissue processor (Excelsior ES, Thermo Scientific, Burlington, ON, Canada). Sections ( $5 \mu \mathrm{m})$ were prepared using an ultramicrotome (Leica Microsystems, Richmond Hill, ON, Canada). Sections were deparaffinised and rehydrated before staining with $\mathrm{H} \& \mathrm{E}$. 


\section{Statistical analysis}

GraphPad Prism 8 statistical software (San Diego, CA, USA) was used to calculate EC50s, perform unpaired $t$-test (two-tailed $p$ values), two-way ANOVA with Sidak's multiple comparisons test, and ordinary one-way ANOVA with Tukey's multiple comparisons test.

\section{Code availability}

DyNeMo algorithm was generated previously [21].

Acknowledgements We thank Brenda Gallie for sharing retinoblastoma cell lines, Yaroslav Fedyshyn for preparing shRNA pools, Michael Parsons at the LTRI Flow Core Facility for help with ImageStream.

Funding This work was funded by grants to RB from the Canadian Institutes for Health Research (CIHR) Grant \#153128, The Cancer Research Society, and the Krembil Foundation.

\section{Compliance with ethical standards}

Conflict of interest The authors declare that they have no conflict of interest.

Publisher's note Springer Nature remains neutral with regard to jurisdictional claims in published maps and institutional affiliations.

Open Access This article is licensed under a Creative Commons Attribution 4.0 International License, which permits use, sharing, adaptation, distribution and reproduction in any medium or format, as long as you give appropriate credit to the original author(s) and the source, provide a link to the Creative Commons license, and indicate if changes were made. The images or other third party material in this article are included in the article's Creative Commons license, unless indicated otherwise in a credit line to the material. If material is not included in the article's Creative Commons license and your intended use is not permitted by statutory regulation or exceeds the permitted use, you will need to obtain permission directly from the copyright holder. To view a copy of this license, visit http://creativecommons. org/licenses/by/4.0/.

\section{References}

1. Dimaras H, Corson TW, Cobrinik D, White A, Zhao J, Munier FL. et al. Retinoblastoma. Nat Rev Dis Prim. 2015;1:15021.

2. Corson TW, Gallie BL. One hit, two hits, three hits, more? Genomic changes in the development of retinoblastoma. Genes Chromosom Cancer. 2007;46:617-34.

3. Rushlow DE, Mol BM, Kennett JY, Yee S, Pajovic S, Thériault $\mathrm{BL}$, et al. Characterisation of retinoblastomas without RB1 mutations: genomic, gene expression, and clinical studies. Lancet Oncol. 2013; 14:327-34.

4. Munier FL, Gaillard M-C, Balmer A, Soliman S, Podilsky G, Moulin AP, et al. Intravitreal chemotherapy for vitreous disease in retinoblastoma revisited: from prohibition to conditional indications. Br J Ophthalmol. 2012;96:1078-83.

5. Francis JH, Abramson DH, Gaillard M-C, Marr BP, BeckPopovic M, Munier FL. The classification of vitreous seeds in retinoblastoma and response to intravitreal melphalan. Ophthalmology. 2015;122:1173-9.

6. De Francesco S, Galluzzi P, Bracco S, Menicacci F, Motolese E, Hadjistilianou T. Alternated intra-arterial and intravitreal chemotherapy for advanced intraocular retinoblastoma: preliminary successful results without systemic chemotherapy. Int Ophthalmol. 2015;35:887-95.

7. Liang T-Y, Zhu X-Y, Hua X-M, Ji X-D, Zhao P-Q. Combined intra-arterial chemotherapy and intravitreal melphalan for the treatment of advanced unilateral retinoblastoma. Int J Ophthalmol. 2020;13:257-62.

8. Francis JH, Iyer S, Gobin YP, Brodie SE, Abramson DH. Retinoblastoma vitreous seed clouds (Class 3): a comparison of treatment with ophthalmic artery chemosurgery with or without intravitreous and periocular chemotherapy. Ophthalmology. 2017; 124:1548-55.

9. Shields CL, Manjandavida FP, Arepalli S, Kaliki S, Lally SE, Shields JA. Intravitreal melphalan for persistent or recurrent retinoblastoma vitreous seeds: preliminary results. JAMA Ophthalmol. 2014;132:319-25.

10. Xu XL, Fang Y, Lee TC, Forrest D, Gregory-Evans C, Almeida $\mathrm{D}$, et al. Retinoblastoma has properties of a cone precursor tumor and depends upon cone-specific MDM2 signaling. Cell. 2009; 137:1018-31.

11. Laurie NA, Donovan SL, Shih C-S, Zhang J, Mills N, Fuller C, et al. Inactivation of the p53 pathway in retinoblastoma. Nature. 2006;444:61-6.

12. Qi D-L, Cobrinik D. MDM2 but not MDM4 promotes retinoblastoma cell proliferation through p53-independent regulation of MYCN translation. Oncogene. 2017;36:1760-9.

13. Laurie NA, Shih C-S, Schin-Shih C, Dyer MA. Targeting MDM2 and MDMX in retinoblastoma. Curr Cancer Drug Targets. 2007;7:689-95.

14. Elison JR, Cobrinik D, Claros N, Abramson DH, Lee TC. Small molecule inhibition of HDM2 leads to p53-mediated cell death in retinoblastoma cells. Arch Ophthalmol. 2006;124:1269-75.

15. Chu WK, Law KS, Chan SO, Yam JCS, Chen LJ, Zhang H, et al. Antagonists of growth hormone-releasing hormone receptor induce apoptosis specifically in retinoblastoma cells. Proc Natl Acad Sci USA. 2016;113:14396-401.

16. Wang H, Bauzon F, Ji P, Xu X, Sun D, Locker J, et al. Skp2 is required for survival of aberrantly proliferating Rb1-deficient cells and for tumorigenesis in Rb1+/- mice. Nat Genet. 2010;42:83-8.

17. Aubry A, Yu T, Bremner R. Preclinical studies reveal MLN4924 is a promising new retinoblastoma therapy. Cell Death Discov. 2020;6:1-12.

18. Moffat J, Grueneberg DA, Yang X, Kim SY, Kloepfer AM, Hinkle $\mathrm{G}$, et al. A lentiviral RNAi library for human and mouse genes applied to an arrayed viral high-content screen. Cell. 2006;124:1283-98.

19. Downward J. Use of RNA interference libraries to investigate oncogenic signalling in mammalian cells. Oncogene. 2004;23: 8376-83.

20. Schuster A, Erasimus H, Fritah S, Nazarov PV, van Dyck E, Niclou SP, et al. RNAi/CRISPR screens: from a pool to a valid hit. Trends Biotechnol. 2019;37:38-55.

21. Taylor IW, Linding R, Warde-Farley D, Liu Y, Pesquita C, Faria $\mathrm{D}$, et al. Dynamic modularity in protein interaction networks predicts breast cancer outcome. Nat Biotechnol. 2009;27:199-204.

22. Lasorella A, Noseda M, Beyna M, Yokota Y, Iavarone A. Id 2 is a retinoblastoma protein target and mediates signalling by Myc oncoproteins. Nature. 2000;407:592-8.

23. Lasorella A, Rothschild G, Yokota Y, Russell RG, Iavarone A. Id 2 mediates tumor initiation, proliferation, and angiogenesis in Rb mutant mice. Mol Cell Biol. 2005;25:3563-74. 
24. Lasorella A, Boldrini R, Dominici C, Donfrancesco A, Yokota Y, Inserra A, et al. Id 2 is critical for cellular proliferation and is the oncogenic effector of $\mathrm{N}$-myc in human neuroblastoma. Cancer Res. 2002;62:301-6.

25. Landreville S, Ma D, Wu J, Harbour JW. Loss of Id2 potentiates the tumorigenic effect of $\mathrm{Rb}$ inactivation in a mouse model of retinoblastoma. Curr Eye Res. 2010;35:435-9.

26. Ceccaldi R, Rondinelli B, D'Andrea AD. Repair pathway choices and consequences at the double-strand break. Trends Cell Biol. 2016;26:52-64

27. Dizin E, Gressier C, Magnard C, Ray H, Décimo D, Ohlmann T, et al. BRCA1 interacts with poly(A)-binding protein: implication of BRCA1 in translation regulation. J Biol Chem. 2006;281: 24236-46.

28. Banerjee R, Russo N, Liu M, Basrur V, Bellile E, Palanisamy N, et al. TRIP13 promotes error-prone nonhomologous end joining and induces chemoresistance in head and neck cancer. Nat Commun. 2014;5:4527.

29. Clairmont CS, Sarangi P, Ponnienselvan K, Galli LD, Csete I, Moreau L, et al. TRIP13 regulates DNA repair pathway choice through REV7 conformational change. Nat Cell Biol. 2020;22:87-96.

30. Vélez-Cruz R, Manickavinayaham S, Biswas AK, Clary RW, Premkumar T, Cole F, et al. RB localizes to DNA double-strand breaks and promotes DNA end resection and homologous recombination through the recruitment of BRG1. Genes Dev. 2016;30:2500-12.

31. Cook R, Zoumpoulidou G, Luczynski MT, Rieger S, Moquet J, Spanswick VJ, et al. Direct involvement of retinoblastoma family proteins in DNA repair by non-homologous end-joining. Cell Rep. 2015;10:2006-18.

32. Yang Y, Tian S, Brown B, Chen $\mathrm{P}, \mathrm{Hu} \mathrm{H}, \mathrm{Xia} \mathrm{L}$, et al. The Rb1 gene inhibits the viability of retinoblastoma cells by regulating homologous recombination. Int J Mol Med. 2013;32:137-43.

33. Marshall AE, Roes MV, Passos DT, DeWeerd MC, Chaikovsky AC, Sage J, et al. RB1 deletion in RB-pathway disrupted cells results in DNA damage and cancer progression. Mol Cell Biol. 2019. https://doi.org/10.1128/MCB.00105-19.

34. Pickering MT, Kowalik TF. Rb inactivation leads to E2F1mediated DNA double-strand break accumulation. Oncogene. 2006;25:746-55.

35. Knudsen ES, Sexton CR, Mayhew CN. Role of the retinoblastoma tumor suppressor in the maintenance of genome integrity. Curr Mol Med. 2006;6:749-57.

36. Thangavel C, Boopathi E, Ciment S, Liu Y, O’Neill R, Sharma A, et al. The retinoblastoma tumor suppressor modulates DNA repair and radioresponsiveness. Clin Cancer Res. 2014;20:5468-82.

37. Garsed DW, Alsop K, Fereday S, Emmanuel C, Kennedy CJ, Etemadmoghadam D, et al. Homologous recombination DNA repair pathway disruption and retinoblastoma protein loss are associated with exceptional survival in high-grade serous ovarian cancer. Clin Cancer Res. 2018;24:569-80.

38. Deng C-X. Roles of BRCA1 in centrosome duplication. Oncogene. 2002;21:6222-7.

39. Zhu Q, Pao GM, Huynh AM, Suh H, Tonnu N, Nederlof PM, et al. BRCA1 tumour suppression occurs via heterochromatinmediated silencing. Nature. 2011;477:179-84.

40. Gorrini C, Baniasadi PS, Harris IS, Silvester J, Inoue S, Snow B, et al. BRCA1 interacts with $\mathrm{Nrf} 2$ to regulate antioxidant signaling and cell survival. J Exp Med. 2013;210:1529-44.

41. Blackshaw S, Harpavat S, Trimarchi J, Cai L, Huang H, Kuo WP, et al. Genomic analysis of mouse retinal development. PLoS Biol. 2004;2:E247.

42. Zhou B-BS, Bartek J. Targeting the checkpoint kinases: chemosensitization versus chemoprotection. Nat Rev Cancer. 2004;4:216-25.
43. Jin $\mathrm{P}, \mathrm{Gu} \mathrm{Y}$, Morgan DO. Role of inhibitory CDC2 phosphorylation in radiation-induced G2 arrest in human cells. J Cell Biol. 1996;134:963-70.

44. Donzelli M, Draetta GF. Regulating mammalian checkpoints through Cdc25 inactivation. EMBO Rep. 2003;4:671-7.

45. Bouwman P, Jonkers J. The effects of deregulated DNA damage signalling on cancer chemotherapy response and resistance. Nat Rev Cancer. 2012;12:587-98.

46. Helleday T, Petermann E, Lundin C, Hodgson B, Sharma RA. DNA repair pathways as targets for cancer therapy. Nat Rev Cancer. 2008;8:193-204.

47. Gavande NS, VanderVere-Carozza PS, Hinshaw HD, Jalal SI, Sears CR, Pawelczak KS, et al. DNA repair targeted therapy: the past or future of cancer treatment? Pharmacol Ther. 2016;160: $65-83$.

48. Abramson DH, Shields CL, Munier FL, Chantada GL. Treatment of retinoblastoma in 2015: agreement and disagreement. JAMA Ophthalmol. 2015;133:1341-7.

49. Tsui JY, Dalgard C, Van Quill KR, Lee L, Grossniklaus HE, Edelhauser HF, et al. Subconjunctival topotecan in fibrin sealant in the treatment of transgenic murine retinoblastoma. Investig Ophthalmol Vis Sci. 2008;49:490-6.

50. Nam C, Doi K, Nakayama H. Etoposide induces G2/M arrest and apoptosis in neural progenitor cells via DNA damage and an ATM/p53-related pathway. Histol Histopathol. 2010;25:485-93.

51. Huang F, Motlekar NA, Burgwin CM, Napper AD, Diamond SL, Mazin AV. Identification of specific inhibitors of human RAD51 recombinase using high-throughput screening. ACS Chem Biol. 2011;6:628-35.

52. Huang F, Mazin AV. A small molecule inhibitor of human RAD51 potentiates breast cancer cell killing by therapeutic agents in mouse xenografts. PLoS ONE. 2014;9:e100993.

53. Chou T-C. Theoretical basis, experimental design, and computerized simulation of synergism and antagonism in drug combination studies. Pharmacol Rev. 2006;58:621-81.

54. Miyashita T, Reed JC. Tumor suppressor p53 is a direct transcriptional activator of the human bax gene. Cell. 1995;80:293-9.

55. Suvarna V, Singh V, Murahari M. Current overview on the clinical update of Bcl-2 anti-apoptotic inhibitors for cancer therapy. Eur J Pharmacol. 2019;862:172655.

56. Lemaître S, Poyer F, Fréneaux P, Leboucher S, Doz F, Cassoux $\mathrm{N}$, et al. Low retinal toxicity of intravitreal carboplatin associated with good retinal tumour control in transgenic murine retinoblastoma. Clin Exp Ophthalmol. 2019. https://doi.org/10.1111/ ceo. 13711

57. Camp DA, Lally SE, Shields CL. Heterochromia following intravitreal chemotherapy in two cases. J AAPOS. 2019. https:// doi.org/10.1016/j.jaapos.2019.03.002.

58. Abbas T, Dutta A. p21 in cancer: intricate networks and multiple activities. Nat Rev Cancer. 2009;9:400-14.

59. Suzuki A, Tsutomi Y, Yamamoto N, Shibutani T, Akahane K. Mitochondrial regulation of cell death: mitochondria are essential for procaspase 3-p21 complex formation to resist Fas-mediated cell death. Mol Cell Biol. 1999;19:3842-7.

60. Tse C, Shoemaker AR, Adickes J, Anderson MG, Chen J, Jin S, et al. ABT-263: a potent and orally bioavailable Bcl-2 family inhibitor. Cancer Res. 2008;68:3421-8.

61. Foucquier J, Guedj M. Analysis of drug combinations: current methodological landscape. Pharmacol Res Perspect. 2015;3: e00149.

62. Rasmussen RD, Gajjar MK, Tuckova L, Jensen KE, MayaMendoza A, Holst CB, et al. BRCA1-regulated RRM2 expression protects glioblastoma cells from endogenous replication stress and promotes tumorigenicity. Nat Commun. 2016;7:13398.

63. Etemadmoghadam D, Weir BA, Au-Yeung G, Alsop K, Mitchell $\mathrm{G}$, George J, et al. Synthetic lethality between CCNE1 
amplification and loss of BRCA1. Proc Natl Acad Sci USA. 2013;110:19489-94.

64. Liu JC, Granieri L, Shrestha M, Wang D-Y, Vorobieva I, Rubie EA, et al. Identification of CDC25 as a common therapeutic target for triple-negative breast cancer. Cell Rep. 2018;23:112-26.

65. Gong X, Du J, Parsons SH, Merzoug FF, Webster Y, Iversen PW, et al. Aurora A kinase inhibition is synthetic lethal with loss of the RB1 tumor suppressor gene. Cancer Discov. 2019;9:248-63.

66. Oser MG, Fonseca R, Chakraborty AA, Brough R, Spektor A, Jennings RB, et al. Cells lacking the RB1 tumor suppressor gene are hyperdependent on aurora B kinase for survival. Cancer Discov. 2019;9:230-47.

67. Corella AN, Cabiliza Ordonio MVA, Coleman I, Lucas JM, Kaipainen A, Nguyen HM, et al. Identification of therapeutic vulnerabilities in small cell neuroendocrine prostate cancer. Clin Cancer Res. 2019. https://doi.org/10.1158/1078-0432.CCR-19-0775.

68. Rudin CM, Hann CL, Garon EB, Ribeiro de Oliveira M, Bonomi PD, Camidge DR, et al. Phase II study of single-agent navitoclax (ABT-263) and biomarker correlates in patients with relapsed small cell lung cancer. Clin Cancer Res. 2012;18:3163-9.

69. Lochmann TL, Floros KV, Naseri M, Powell KM, Cook W, March RJ, et al. Venetoclax is effective in small-cell lung cancers with high BCL-2 expression. Clin Cancer Res. 2018;24:360-9.

70. Maclay T, Day M, Mills K. Abstract 363: CYT01B, a novel RAD51 inhibitor, acts synergistically with PARP inhibitors. Cancer Res. 2019;79:363.

71. FitzSimmons J, Fantel A, Shepard TH. Growth parameters in midtrimester fetal Turner syndrome. Early Hum Dev. 1994;38:121-9.

72. Shepard TH. Growth and development of human embryo and fetus. Philadelphia: W.B. Saunders; 1975.

73. Chen D, Livne-bar I, Vanderluit JL, Slack RS, Agochiya M, Bremner R. Cell-specific effects of RB or RB/p107 loss on retinal development implicate an intrinsically death-resistant cell-oforigin in retinoblastoma. Cancer Cell. 2004;5:539-51.

74. Sangwan M, McCurdy SR, Livne-Bar I, Ahmad M, Wrana JL, Chen D, et al. Established and new mouse models reveal E2f1 and Cdk2 dependency of retinoblastoma, and expose effective strategies to block tumor initiation. Oncogene. 2012;31:5019-28.

75. MacPherson D, Sage J, Kim T, Ho D, McLaughlin ME, Jacks T. Cell type-specific effects of $\mathrm{Rb}$ deletion in the murine retina. Genes Dev. 2004;18:1681-94.

76. Pajovic S, Corson TW, Spencer C, Dimaras H, Orlic-Milacic M, Marchong MN, et al. The TAg-RB murine retinoblastoma cell of origin has immunohistochemical features of differentiated Muller glia with progenitor properties. Investig Ophthalmol Vis Sci. 2011;52:7618-24.

77. Windle JJ, Albert DM, O'Brien JM, Marcus DM, Disteche CM, Bernards R, et al. Retinoblastoma in transgenic mice. Nature. 1990;343:665-9.

78. Sims D, Mendes-Pereira AM, Frankum J, Burgess D, Cerone MA, Lombardelli $\mathrm{C}$, et al. High-throughput RNA interference screening using pooled shRNA libraries and next generation sequencing. Genome Biol. 2011;12:R104.

79. To K-H, Pajovic S, Gallie BL, Thériault BL. Regulation of p14ARF expression by miR-24: a potential mechanism compromising the p53 response during retinoblastoma development. BMC Cancer. 2012;12:69.

80. Anderson SN, Towne DL, Burns DJ, Warrior U. A highthroughput soft agar assay for identification of anticancer compound. J Biomol Screen. 2007;12:938-45.

81. Chen D, Pacal M, Wenzel P, Knoepfler PS, Leone G, Bremner R. Division and apoptosis of E2f-deficient retinal progenitors. Nature. 2009;462:925-9. 